\title{
SPIRou: nIR velocimetry \& spectropolarimetry at the CFHT
}

\author{
J.-F. Donati ${ }^{1 \star}$, D. Kouach ${ }^{2}$, C. Moutou1 ${ }^{1}$, R. Doyon ${ }^{3}$, X. Delfosse ${ }^{4}$, E.Artigau ${ }^{3}$, \\ S. Baratchart ${ }^{2}$, M. Lacombe ${ }^{2}$, G. Barrick ${ }^{5}$, G. Hébrard ${ }^{6}$, F. Bouchy ${ }^{7,8}$, L. Saddlemyer ${ }^{9}$, \\ L. Parès ${ }^{1}$, P. Rabou ${ }^{4}$, Y. Micheau ${ }^{2}$, F. Dolon ${ }^{10}$, V. Reshetov ${ }^{9}$, Z. Challita ${ }^{2}$,
} A. Carmona ${ }^{1,4}$, N. Striebig ${ }^{2}$, S. Thibault ${ }^{11}$, E. Martioli ${ }^{12,6}$, N. Cook ${ }^{3}$, P. Fouquée, ${ }^{5,1}$, T. Vermeulen ${ }^{5}$, S.Y. Wang ${ }^{13}$, L. Arnold ${ }^{5,10}$, F. Pepe ${ }^{7}$, I. Boisse ${ }^{8}$, P. Figueira ${ }^{14,15}$, J. Bouvier ${ }^{4}$, T.P. Ray ${ }^{16}$, C. Feugeade ${ }^{1}$, J. Morin ${ }^{17}$, S. Alencar ${ }^{18}$, M. Hobson ${ }^{8}$, B. Castilho ${ }^{12}$, S. Udry ${ }^{7}$, N.C. Santos ${ }^{15}$, O. Hernandez ${ }^{3,19}$, T. Benedict ${ }^{5}$, P. Vallée ${ }^{3}$, G. Gallou ${ }^{1}$, M. Dupieux ${ }^{1}$, M. Larrieu ${ }^{1}$, S. Perruchot ${ }^{10}$, R. Sottile ${ }^{10}$, F. Moreau ${ }^{10}$, C. Usher ${ }^{5}$, M. Baril ${ }^{5}$, F. Wildi ${ }^{7}$, B. Chazelas ${ }^{7}$, L. Malo $^{3}$, X. Bonfils ${ }^{4}$, D. Loop ${ }^{9}$, D. Kerley ${ }^{9}$, I. Wevers ${ }^{9}$, J. Dunn ${ }^{9}$, J. Pazder ${ }^{9}$, S. Macdonald ${ }^{9}$, B. Dubois ${ }^{2}$, E. Carrié ${ }^{2}$, H. Valentin ${ }^{1}$, F. Henault ${ }^{4}$, C.H. Yan ${ }^{13}$, T. Steinmetz ${ }^{20}$

Affiliations are listed at the end of the paper

Accepted 2020 August 19. Received 2020 August 18; in original form 2020 May 12

\begin{abstract}
This paper presents an overview of SPIRou, the new-generation near-infrared spectropolarimeter / precision velocimeter recently installed on the 3.6-m Canada-FranceHawaii Telescope (CFHT). Starting from the two main science goals, namely the quest for planetary systems around nearby $\mathrm{M}$ dwarfs and the study of magnetized star / planet formation, we outline the instrument concept that was designed to efficiently address these forefront topics, and detail the in-lab and on-sky instrument performances measured throughout the intensive testing phase that SPIRou was submitted to before passing the final acceptance review in early 2019 and initiating science observations. With a central position among the newly started programmes, the SPIRou Legacy Survey (SLS) Large Programme was allocated 300 CFHT nights until at least mid 2022.

We also briefly describe a few of the first results obtained in the various science topics that SPIRou started investigating, focusing in particular on planetary systems of nearby $\mathrm{M}$ dwarfs, transiting exoplanets and their atmospheres, magnetic fields of young stars, but also on alternate science goals like the atmospheres of $\mathrm{M}$ dwarfs and the Earth's atmosphere. We finally conclude on the essential role that SPIRou and the CFHT can play in coordination with forthcoming major facilities like the JWST, the ELTs, PLATO and ARIEL over the decade.
\end{abstract}

Key words: stars: planetary systems - stars: formation - stars: magnetic fields techniques: radial velocities - instrumentation: polarimeters - instrumentation: spectrographs 
scopes have been quite successful at obtaining forefront results in various research fields relevant to the physics of stars and planets. In particular, HARPS on the 3.6-m European Southern Observatory (ESO) telescope (Mayor et al. 2003 ) with its exquisite sub- $\mathrm{m} \mathrm{s}^{-1}$ velocimetric precision has been instrumental in unveiling and characterizing hundreds of planetary systems orbiting stars other than the Sun (e.g., Mayor 2019); similarly, ESPaDOnS on the 3.6-m CanadaFrance-Hawaii Telescope (CFHT, Donati 2003) revolutionized the field of stellar magnetometry thanks to its polarimetric capabilities (Donati \& Landstreet 2009), by revealing and mapping the surface magnetic fields in stars of all types, including newborn stars where magnetic fields are known to play a key role (e.g., Bouvier 2017; Pudritz \& Ray 2019). More recently, new-generation velocimeters (like ESPRESSO on the ESO $4 \times 8-\mathrm{m}$ Very Large Telescope / VLT, Pepe et al. 2013) are pushing further into the $\mathrm{dm} \mathrm{s}^{-1}$ precision regime to unveil Earth-like planets located in the habitable zone (HZ) of their host stars, i.e., in the adequate distance range from the star for liquid water to be able to pool on the planet surface.

To deepen this exploration further and reach yet new classes of stars, in particular very-low-mass dwarfs and very young class-I protostars currently out of reach of existing facilities given their intrinsic faintness at visible wavelengths, new instruments working in the near infrared (nIR) were designed and constructed. These include GIANO on the $3.6-\mathrm{m}$ Telescopio Nazionale Galileo (Oliva et al. 2018), Carmenes on the 3.5-m Telescope at Calar Alto (Quirrenbach et al. 2012), iSHELL on the 3.2-m NASA InfraRed Telescope Facility (Rayner et al. 2016), HPF on the 10-m Hobby-Ebberly Telescope (Mahadevan et al. 2014), IRD on the 8-m Subaru Telescope (Kotani et al. 2014, 2018) and soon NIRPS on the 3.6-m ESO telescope (Bouchy et al. 2019). On-sky operations have started in the last few years for most of these instruments, often aiming at extensive velocimetric surveys of nearby $\mathrm{M}$ dwarfs. This exploration represents a critical step in our long-term quest to understand the emergence of life, by paving the ground for future scrutinizing studies of the atmospheres of the best Earth-like planet candidates with large facilities such as the 6-m James Webb Space Telescope (JWST), the Extremely Large Telescopes (ELTs ${ }^{1}$, e.g., Cirasuolo 2019), and later-on the ARIEL space probe (Tinetti et al. 2017).

SPIRou (standing for SpectroPolarimètre InfraRouge, i.e., Infrared SpectroPolarimeter, Donati et al. 2018) is one of these new instruments, designed and constructed for the CFHT, and inspired from both HARPS and ESPaDOnS. Covering the whole YJHK bands $(0.95-2.5 \mu \mathrm{m})$ in a single exposure, SPIRou includes an achromatic polarimeter fiberfeeding a cryogenic high-resolution échelle spectrograph capable of both high-precision velocimetry and spectropolarimetry of stars. The two main science goals that motivated the construction of SPIRou are the quest for planetary systems around nearby $M$ dwarfs on the one hand, and the study of magnetized star / planet formation on the other; this core programme, called the SPIRou Legacy Survey (SLS), was allocated 300 CFHT nights from 2019 until

1 Including the 39-m ESO's ELT, the Thirty Meter Telescope / TMT, and the 25-m Giant Magellan Telescope / GMT at least mid 2022. Whereas precision velocimetry is key for the first goal, spectropolarimetry is required for the other and can yield at the same time optimal proxies for monitoring activity in the surveyed M dwarfs (Hébrard et al. 2016; Haywood et al. 2016). Beyond these two main goals, SPIRou can efficiently explore a wide variety of science topics, from planetary atmospheres (both for Solar-System planets and exoplanets) to weather patterns of brown dwarfs, stellar dynamos, stellar archaeology and seasonal variations in the Earth's atmosphere.

In this paper, we start by detailing the science goals of SPIRou (in Sec. 2), describe its technical characteristics (in Sec. 3), its measured performances (in Sec. 4), and present a short overview of the first results so far, mostly within the SLS (in Sec. 5). We finally conclude by outlining how the potential of SPIRou can be best exploited in the coming decade and beyond, in particular to prepare and contribute to future explorations to be carried out with planned major facilities from both ground and space, including the JWST, the ELTs, PLATO and ARIEL (in Sec. 6).

\section{SPIROU SCIENCE : MAIN GOALS \& COMPLEMENTARY OBJECTIVES}

We start this overall description of SPIRou by outlining the two main science goals for which SPIRou was designed and on which the SLS is focused, as well as the complementary objectives that SPIRou can tackle. We also present the international science consortium involved in the funding and construction of SPIRou.

\subsection{Planetary systems of $M$ dwarfs}

In the quest for worlds other than the Solar System, in particular those harbouring Earth-like planets located in the $\mathrm{HZ}$ of their host stars, $\mathrm{M}$ dwarfs have attracted tremendous interest over the last few years (e.g., Bonfils et al. 2013; Muirhead et al. 2015). In addition to being the most populated stellar class in the solar neighbourhood, $\mathrm{M}$ dwarfs were shown to be planet rich (especially in low-mass planets), hosting on average $>2$ planets per star (e.g., Dressing \& Charbonneau 2015; Gaidos et al. 2016; Gillon et al. 2017) with a large fraction of them located in the HZ (Bonfils et al. 2013). In this context, studying planetary system architectures of $\mathrm{M}$ dwarfs and unveiling detailed statistics on the planet occurrence rates come as obvious goals for investigating the extent to which the masses of the host stars (and thereby the properties of the parent protoplanetary disc) impact star / planet formation. Moreover, future atmospheric studies with, e.g, the JWST, the ELTs or ARIEL, of all HZ Earth-like planets to be detected and characterized through transit photometry and velocimetry will only be possible for those orbiting the nearest stars, which happen to be $\mathrm{M}$ dwarfs.

As of now, the number of known planets and systems in the solar neighbourhood (within, say, $10 \mathrm{pc}$ ) is quite limited, of order $10 \%$ of the actual planet population predicted from what we know of planetary systems of early-M dwarfs; for 
instance, only about 35 planets around 19 stars $^{2}$ have yet been identified among the $140 \mathrm{M}$ dwarfs within $6.6 \mathrm{pc}$ of the Sun, which should together host a total of 310 planets (assuming an average of 2.2 planets per $\mathrm{M}$ dwarf, Gaidos et al. 2016). One of the reasons for this relative ignorance is that few high-precision velocimeters were, until recently, capable of detecting and characterizing planetary systems around $\mathrm{M}$ dwarfs, especially for late-type ones, thereby significantly limiting the accessible sample; being carried out with visible spectrographs, surveys concentrating on $\mathrm{M}$ dwarfs have indeed always been photon-noise limited at a radial velocity (RV) precision worse than $1 \mathrm{~m} \mathrm{~s}^{-1}$ (e.g., Bonfils et al. 2013), and as such severely limited our ability to detect planets. Another reason is that a proper characterization of the planet masses in multi-planet systems require a large number of visits, typically a few hundreds, in order to disentangle the contributions of all planets (e.g., Udry et al. 2019), even when the planet periods are known from transit photometry (e.g., Cloutier et al. 2019). Last but not least, M dwarfs are on average more active than the Sun, making it harder to accurately determine the planet masses if stellar activity is not faithfully modeled, which requires a dense and regular monitoring of the host star (e.g., Klein \& Donati 2019).

SPIRou and the SLS tackle this goal in two complementary ways, through (i) a systematic RV monitoring of about 70 nearby $\mathrm{M}$ dwarfs (with an average of about 150 visits per star and a special emphasis on planets lying within HZs), called the SLS Planet Search (SLS-PS), and (ii) a RV follow-up of about 20 of the most interesting transiting planet candidates to be uncovered by photometric surveys (with an average of about 40 visits per star), called the SLS Transit Follow-up (SLS-TF). Each visit provides a spectrum with a signal to noise ratio (SNR) of 250 to 150 per pixel (for early-M to late-M dwarfs respectively), expected to yield a photon-noise RV precision of $\simeq 1 \mathrm{~m} \mathrm{~s}^{-1}$, with spectropolarimetry being used in most cases to simultaneously monitor magnetic activity. With average $\mathrm{H}$ magnitudes of 7 and 9 respectively, SLS-PS and SLS-TF targets require typical exposure times of about $6 \mathrm{~min}$ and $40 \mathrm{~min}$ per visit.

The main goal of the SLS-PS is to unveil and characterize (i.e., measure the orbital parameters and derive lower limits on the masses of) a large fraction of the still unknown planets in the immediate neighborhood of the Solar System, in order to achieve a large census and statistical study of the planet population around nearby $\mathrm{M}$ dwarfs (especially the less-explored lowest-mass stars) and thereby make a major step forward in our understanding of how systems like ours form and evolve into maturity. SLS-PS observations will yield an accurate measurement of the occurrence of Earth-like planets orbiting mid- to late-M dwarfs, especially those located within the HZ, to be compared with prior estimates from HARPS and Kepler data in the specific case of early-M dwarfs. From Monte-Carlo simulations, we estimate that the SLS-PS, totalling 150 CFHT nights ( $50 \%$ of the SLS allocation) should detect $\simeq 60$ new exoplan-

\footnotetext{
2 Namely Gl 15A, GJ 54.1=YZ Cet, GJ 1061, GJ 191=Kapteyn's star, GJ 3323, Gl 229, GJ 273, GJ 338B, Gl 411, Gl 447=Ross 128, GJ 551=Proxima Cen, Gl 581, Gl 625, Gl 628, Gl 674, Gl 687, Gl 752, Gl 832 and GJ 876 according to the NASA exoplanet catalog at https://exoplanetarchive.ipac.caltech.edu.
}

ets (yielding an average planet detection efficiency of $\simeq 40 \%$ assuming 2.2 planets per star, in line with Cloutier et al. 2018), including $\simeq 25$ Earth-mass planets ( $\simeq 6$ of which located in the HZ of their host stars). The SLS-PS will also serve as a pioneering exploration to pin down the closest and very best planet candidates for future detailed characterizations of their atmospheres with the JWST and eventually the ELTs (including for non-transiting planets, by combining high-dispersion spectroscopy with high-contrast imaging, Snellen et al. 2015). The SLS-PS input catalog, based on preparatory observations with ESPaDOnS at the CFHT, includes a list of all potential targets ranked according to a merit function taking into account several parameters including brightness and rotation rate, or equivalently activity level (Moutou et al. 2017; Fouqué et al. 2018).

In the case of the SLS-TF to which 75 CFHT nights ( $25 \%$ of the SLS allocation) are dedicated, the main goals are (i) to constrain the mass-radius diagram of low mass planets that will revolutionize our understanding of their internal structure and bulk composition and (ii) to provide key mass measurements of the best planets amenable to atmospheric characterization with SPIRou through high-resolution transit spectroscopy (Brogi et al. 2018; Brogi \& Line 2019) and later-on with the JWST, the ELTs and ARIEL. The bulk of the SLS-TF targets to be monitored is provided by TESS (Ricker et al. 2016). Knowing in advance both orbital periods and transit times from photometry partly compensates for SLS-TF targets being in average 2 magnitudes fainter than SLS-PS ones and ensures that the velocimetric signal can be detected with only a few tens of visits for simple systems, even when activity dominates the RV signal (Klein \& Donati 2020, Klein et al. 2020a, submitted). Among the $\simeq 500$ planets orbiting $\mathrm{M}$ dwarfs that TESS expects to unveil, we will monitor about 20 of the most interesting ones around the brightest stars visible from CFHT, in particular those located in the HZ of their host stars ( $~ 10$ for mid $\mathrm{M}$ dwarfs with $\mathrm{K}<10$, Barclay et al. 2018). The SLS-TF also includes continuous transit monitoring for a few known transiting planets (e.g., HD 189733 b, AU Mic b) to model the Rossiter-McLaughlin effect (Moutou et al. 2020; Martioli et al. 2020) and characterize the properties of the planet atmospheres (Darveau-Bernier et al. 2020; Boucher et al. 2020; Klein et al. 2020b, in prep).

As most SLS observations are using spectropolarimetry, SPIRou data can investigate at the same time the small- and large-scale magnetic properties and the associated activity of the host stars. By modeling the magnetic activity of the sample stars, we not only expect to improve the precision at which RV curves are secured (thanks to filtering techniques using optimal proxies, e.g., Hébrard et al. 2016; Haywood et al. 2016) and thereby enhance the sensitivity to low-mass planets, but also to provide opportunities for characterizing magnetic fields and dynamo processes of largely- and fullyconvective $\mathrm{M}$ dwarfs whose observational properties are still enigmatic (Morin et al. 2008, 2010, 2011; Yadav et al. 2015; Shulyak et al. 2017). Last but not least, this approach will also allow us to study the extent to which magnetic fields and winds of active $\mathrm{M}$ dwarfs can impact the habitability of their HZ planets (Vidotto et al. 2013; Güdel et al. 2014; Strugarek et al. 2015; Vidotto et al. 2019). 


\subsection{Star/planet formation}

Studying how low-mass stars and their planets form and migrate comes as a logical counterpart to the observation of mature planetary systems. More specifically, we aim at investigating the key role that magnetic fields play at all phases of the star/planet formation process (e.g., Königl 1991; André et al. 2009; Bouvier 2017; Pudritz \& Ray 2019; Hennebelle et al. 2020). By controlling accretion, triggering outflows and jets, and producing intense X-rays, magnetic fields indeed critically impact the physics of pre-main sequence (PMS) stars (Baraffe \& Chabrier 2010; Feiden 2016) and of their accretion discs (Shu et al. 2007), and largely dictate their angular momentum evolution (Bouvier et al. 2007). In particular, magnetic fields are thought to couple accreting PMS stars with their discs. At some stage in the process, fields carve magnetospheric gaps in the central disc regions and trigger funneled inflows \& outflows from the inner discs, forcing the host stars to spin down (Romanova et al. 2004, 2011; Zanni \& Ferreira 2013; Davies et al. 2014). Magnetic fields presumably affect planet formation as well (Johansen 2009), can stop or even reverse planet migration (Baruteau et al. 2014), and may prevent close-in planets, including hot-Jupiters (hJs, Lin et al. 1996; Romanova \& Lovelace 2006), from falling into their host stars.

With the Magnetic PMS star/planet survey (SLS-MP), the SLS amplifies the effort initiated on this topic 15 years ago through spectropolarimetric observations of $\mathrm{T}$ Tauri stars (TTSs) and inner protostellar accretion discs of the FUOr type, with ESPaDOnS at the CFHT in particular (e.g., Donati et al. 2005, 2012, 2020; Yu et al. 2017; Hill et al. 2019). This exploratory work led to the characterization of the large-scale magnetic fields of TTSs, to novel constraints about the fossil or dynamo origin of these fields, to the discovery that the magnetic topologies largely reflect the internal structure of PMS stars (Gregory et al. 2012), and to the study of how such fields impact star/planet formation. Along with contemporary photometric data, these observations also revealed that close-in giant planets can indeed already be present around PMS stars at as early an age as a few Myr (David et al. 2016; Donati et al. 2017; Yu et al. 2017; David et al. 2019); these results provide evidence that planet-disc interactions are instrumental in planet formation, likely shaping the early architecture of planetary systems, and thereby yield strong observational constraints on the timescale of planet formation.

To expand this exploration further, the SLS-MP carries out a spectropolarimetric monitoring of about 55 PMS stars in nearby star forming regions, including a handful of very young partly-embedded class-I protostars in which magnetic fields are still mostly unknown (e.g., Flores et al. 2019), with the goal of characterizing these fields, of unveiling the potential presence of close-in giant planets, and ultimately, of investigating in a more systematic fashion the impact of magnetic fields on star/planet formation. As the missing link between the youngest class-0 protostars whose fields are surveyed with ALMA / NOEMA at mm wavelengths (e.g., Maury et al. 2010) and the older TTSs observed with optical instruments like ESPaDOnS, class-I protostars are key for fingerprinting the impact of magnetic fields on star/planet formation, by revealing the topologies of their fields, by telling us how dynamos and magnetospheric accretion be- have when accretion is much stronger and more stochastic than in TTSs, by finding out how large a magnetospheric gap these fields are able to carve at the centre of the disc and how stars react to this process. Given their intrinsic faintness at optical wavelengths (as a result of extinction by the dust cocoon in which they are still embedded), class-I protostars can only be studied at nIR wavelengths, hence the pioneering role that SPIRou and the SLS-MP are expected to play in this task, without suffering from too small a wavelength range in a single exposure like previous unsuccessful studies on such objects (e.g., with CRIRES on the VLT, Viana Almeida et al. 2012).

Besides class-I protostars, the SLS-MP sample features about 50 TTSs, either still surrounded by and actively accreting from their disc (called classical TTSs / cTTSs), or having already dissipated their inner discs (called weak-line TTSs / wTTSs). The SLS-MP includes in particular cTTSs with medium to strong accretion rates and very-low-mass PMS stars for which only few spectropolarimetric observations exist so far, so as to sample the whole range of masses and accretion patterns (Cody et al. 2014; Sousa et al. 2016). Regularly monitoring such cTTSs for several rotation cycles will shed light on their variability, and especially on the dynamics of the star-disk interaction process (e.g., Alencar et al. 2018). Similarly, by monitoring a larger sample of wTTSs than the one already explored (e.g., Yu et al. 2019), the SLS-MP ambitions to characterize the population of newborn close-in giant planets at early evolutionary stages, estimate the occurrence frequency of such planets around PMS stars and compare it with that around mature Sun-like stars; ultimately, one may even detect and estimate the magnetic fields of these hJs using radio observations (Vidotto \& Donati 2017). For all stars, Zeeman-Doppler Imaging (ZDI, e.g., Donati et al. 2020, and references therein) will be used to infer brightness distributions and magnetic topologies at the surfaces of stars, and maps of accretion spots in the accreting PMS stars; by extracting the main properties of the reconstructed large-scale fields, one can then investigate how dynamo processes respond to the evolutionary changes in the structure of PMS stars as they evolve along Hayashi tracks (Gregory et al. 2012; Emeriau-Viard \& Brun 2017; Villebrun et al. 2019).

The SLS-MP aims at securing 20 to 35 visits per star, each visit yielding an intensity and a circularly polarized spectrum with typical SNRs of about 200 per pixel, for a total allocation of 75 CFHT nights (25\% of the SLS allocation). The typical exposure time is $\simeq 20 \mathrm{~min}$, corresponding to an average $\mathrm{H}$ magnitude of 8.3 .

\subsection{Complementary science objectives}

SPIRou can also tackle a large number of additional science programmes, both within the SLS through its Legacy dimension and beyond the two main goals on which the SLS focuses.

By providing a wide and homogeneous set of nIR spectra for a large sample of M dwarfs and PMS stars, the SLS programmes offer the opportunity to assess theoretical atmospheric models of cool and very cool stars in much more detail than currently possible, with the goal of obtaining more precise determinations of the spectroscopic parameters characterizing the observed targets (in particular $T_{\text {eff }}$, 
$\log g$, metallicity, also essential for planet characterization). It makes it possible to further constrain the key physical ingredients (including magnetic fields) playing a role in the atmospheres of very-cool stars and affecting their thermal and convection patterns; such studies will lead to new sets of synthetic spectra that better match nIR observations (Rajpurohit et al. 2013; Allard et al. 2013; Rajpurohit et al. 2018a,b), whereas the use of multi-component model atmospheres will improve the spectroscopic characterization of very active PMS stars (Gully-Santiago et al. 2017).

Studying weather patterns in the atmospheres of brown dwarfs is another particularly exciting option. These objects are known to exhibit photometric variations on short timescales (Artigau et al. 2009), attributed to the presence of atmospheric clouds rotating in and out of view, and subject to temporal variability on timescales of only a few rotation cycles. Using tomographic imaging applied to time-series of high-resolution spectra, one can recover surface maps of the cloud patterns (Crossfield et al. 2014) and potentially their temporal evolution as well for the brightest targets. With its high sensitivity and large spectral domain that can partly compensate for the intrinsic faintness of these very cool objects, SPIRou is a promising instrument for carrying out such studies.

SPIRou is equally well suited for investigating the dynamics and chemistry of planetary atmospheres in our Solar System (Machado et al. 2014, 2017), and potentially of giant close-in exoplanet atmospheres as well, even when not transiting (Snellen et al. 2010; Brogi et al. 2012). Last but not least, SPIRou will also offer the opportunity of studying at high spectral resolution extremely metal-poor stars as relics of the early universe, providing us with precious clues about the chemical evolution and formation of the Milky Way (Reggiani et al. 2016).

Independently of the science programmes they are collected for, SPIRou data can also be used to characterise the chemical content of the Earth's atmosphere, and in particular of the main molecular species such as $\mathrm{H}_{2} \mathrm{O}, \mathrm{CO}_{2}, \mathrm{CH}_{4}$ and $\mathrm{O}_{2}$, through fitting the very rich telluric spectrum fingerprinted in each single SPIRou observations, using tools such as TAPAS, TelFit or Molecfit (Bertaux et al. 2014; Gullikson et al. 2014; Smette et al. 2015; Ulmer-Moll et al. 2019). The extensive collection of spectra that SPIRou will collect over the coming decade provides the community with a novel way of quantitatively investigating the chemical content, as well as the seasonal and yearly evolution, of the Earth's atmosphere over the best astronomical site in the Northern Hemisphere. SPIRou spectra can also be exploited to study $\mathrm{OH}$ and $\mathrm{O}_{2}$ terrestrial night-glow emission lines from the sky, as previously done from optical spectra (Cosby et al. 2006).

\subsection{The SPIRou / SLS science consortium}

The SLS, whose main science topics are outlined above, was allocated 300 observing nights at the CFHT from 2019 to at least mid 2022 (and beyond, until a completion rate of at least $80 \%$ is achieved if not by mid-2022), with the ambitious goal of addressing the following 6 key questions through its multi-faceted inter-connected components (SLS-PS, SLS-TF and SLS-MP):
- How diverse are planetary systems of nearby M dwarfs? How frequently do these stars harbor close-in or HZ Earthmass planets? How does this diversity evolve with the hoststar mass down to the brown-dwarf limit, a mostly unexplored mass range so far?

- How does the density, structure and bulk composition of low-mass transiting planets change with planet mass and stellar irradiation, in particular for the $\mathrm{HZ}$ ones for which very few data exist?

- What are the as-of-yet undetected systems, that are best suited for characterizations, of planetary atmospheres in particular, with future facilities like the JWST and the ELTs?

- What kind of magnetic fields do largely- and fullyconvective $\mathrm{M}$ dwarfs generate as they spin down to low activity levels for which almost no data are available? How do their fields and associated activity impact planet detectability and habitability?

- What types of magnetic fields do low-mass PMS stars (and their accretion discs when relevant) host, in particular the youngest and strongly-accreting stars, for which very few magnetic observations exist? How do these fields change as stars and planets evolve?

- How do such fields impact accretion/ejection processes and star/planet formation, and what is their role in the formation and survival of close-in planets, especially hJs known for their huge dynamic impact on the early architecture of planetary systems?

The SPIRou / SLS science consortium involves about 150 scientists worldwide and includes in particular the core SPIRou science team who proposed the instrument, secured its funding, closely followed its design, construction and validation, and developed optimal tools for data reduction and velocimetric / spectropolarimetric analyses. Deeply rooted in the Franco-Canadian collaboration, born and grown strong and fruitful with the construction and operation of the CFHT, the SLS / SPIRou consortium also gathers collaborators from other CFHT partners (Taiwan, Hawaii) as well as institutes involved in the construction / funding of SPIRou (in Brazil, Switzerland, Portugal and Ireland) from outside the CFHT community.

\section{INSTRUMENT CHARACTERISTICS}

SPIRou is inspired from previous instruments, namely HARPS regarding precision velocimetry and ESPaDOnS regarding spectropolarimetry. It includes a cryogenic highresolution spectrograph adapted from the vacuum-operated spectrograph of HARPS, a Cassegrain unit with an achromatic polarimeter derived from that of ESPaDOnS, a fiber-feed evolved from those of both ESPaDOnS and HARPS, and a calibration / RV reference unit copied from that of HARPS (see Fig. 1 for a CAD view and http://spirou.irap.omp.eu/Gallery2/Photos for pictures).

We describe these units below, and outline the installation and operation of SPIRou at the CFHT. We also briefly describe the SPIRou project team, managed by both Observatoire Midi Pyrénées and the Institut de Recherche en Astrophysique et Planétologie (OMP/IRAP) in Toulouse 


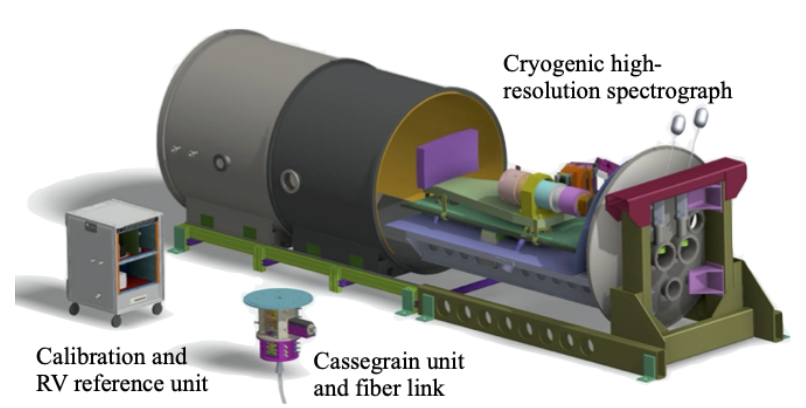

Figure 1. CAD view of the main SPIRou units

(France), and the worldwide institutes involved in the design, construction, tests and operation of SPIRou.

\subsection{Cassegrain unit}

The SPIRou Cassegrain unit consists of 3 structures mounted on top of each other and fixed at the Cassegrain focus of the telescope, for a total height, width and weight of $800 \mathrm{~mm}, 770 \mathrm{~mm}$ and $120 \mathrm{~kg}$. A CAD view of the lower two modules is shown in Fig. 2.

The upper Cassegrain structure essentially serves as a mechanical interface with the telescope, ensuring that the instrument aperture is ideally placed with respect to the telescope focus. It also includes an option for feeding the instrument with a fully polarized beam, allowing one to achieve a complementary polarimetric diagnostic of all optical components above the polarimeter (hence its designation as the "end-to-end calibration module")

The lower Cassegrain structure first includes the instrument aperture, which, instead of being a genuine hole in the middle of a tilted mirror (as for ESPaDOnS and HARPS), consists of a circular region (of diameter 1.29", i.e., $180 \mu \mathrm{m}$ ) located at the centre of a silica plate (called the field mirror) whose lower surface features a reflective coating outside of the central region. As a result, all incoming light falling outside the entrance aperture, as well as a few $\%$ of the light falling inside, is reflected off by the field mirror towards the viewing camera (see below). This structure also includes a focal reducer turning the $\mathrm{f} / 8$ incoming beam entering the aperture, into a $\mathrm{f} / 4$ beam with which the science optical fibers are fed with minimum Focal Ratio Degradation (FRD, see Sec. 3.2). This focal reducer involves a doublet and a triplet working at infinite conjugate ratio, both optimized regarding throughput and image quality over the spectral range of SPIRou. Last but not least, this structure features an achromatic polarimeter, consisting of two 3/4-wave ZnSe dual Fresnel rhombs coupled to a Wollaston prism, splitting the incoming beam into 2 orthogonally polarized beams and feeding the twin science optical fibers (see Sec. 3.2). By tuning the orientation of the rhombs, one can measure the amount of either circular or linear polarization in the incoming stellar light; by coating one of the internal reflection surfaces of each rhomb, we can ensure that the polarimetric analysis is achromatic to better than a few degrees. ZnSe rhombs turned out to be quite tricky to assemble, with molecular adherence between the rhombs being very fragile and requiring the rhomb surface micro-roughness to be better than a few nm not to break under vibrations

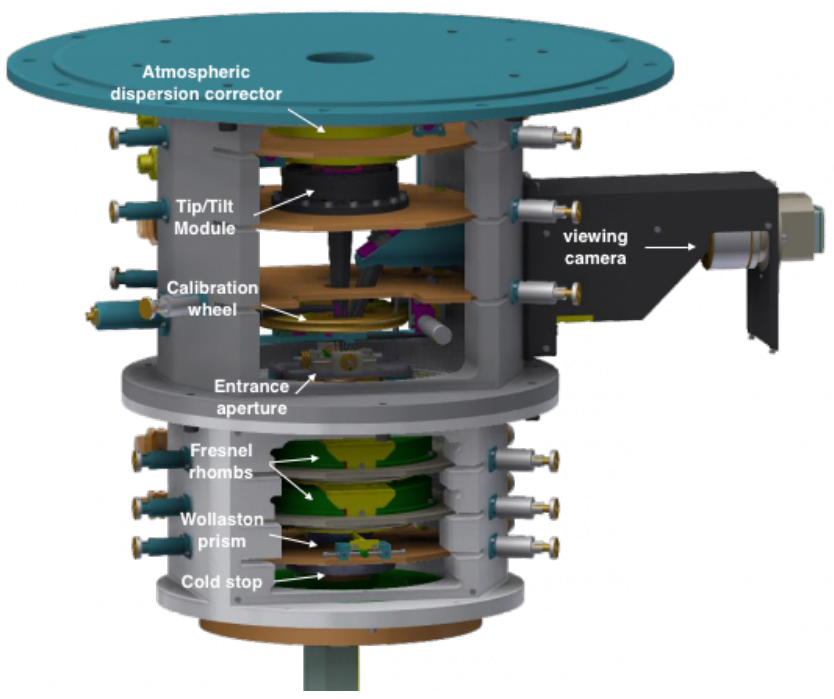

Figure 2. CAD view of the middle and lower structures of the SPIRou Cassegrain unit. The cold stop at the bottom of the lower structure, yielding no improvement in thermal background, was finally not used.

or stress (e.g., induced by temperature variations and gradients); the stress-free mounting of the rhombs into their mechanical barrels also proved complex to master, as was the need to constrain the beam deviation to no more than a few " to minimize the potential RV impact of rotating the rhombs. After several attempts, fabrication challenges were successfully overcome by WinLight Optics (France); the corresponding rhombs, made of ZnSe blanks from a different provider and with higher throughput in the blue, are progressively reaching CFHT, with one already mounted in SPIRou in June 2020 and a few more expected soon.

A cold stop was initially implemented to mask the central pupil obscuration by the telescope secondary mirror and the associated thermal emission, but turned out to bring no significant reduction in the level of thermal background while decreasing the throughput by $\simeq 10 \%$ and was finally not used. Baffles with gold coating are also inserted between the polarimeter optical elements for the same purpose, and in particular between the field mirror and the entrance doublet, and between the triplet and the science fiber head. The thermal background that the polarimeter generates, scaling up with temperature, is about $15 \mathrm{ph} \mathrm{s}^{-1} \AA^{-1}$ per science fiber at $2.35 \mu \mathrm{m}$, for a polarimeter temperature of $2^{\circ} \mathrm{C}$.

The middle Cassegrain structure features several ancillary facilities used for both calibration and observing purposes. It first includes an atmospheric dispersion corrector (ADC) made of two motorized twin-prism pairs cancelling the impact of atmospheric refraction on the incoming beam up to airmasses of 2.5; it ensures that the position of the star on the entrance aperture is achromatic over the full spectral range to a precision better than $0.03^{\prime \prime}$. The middle structure also includes a tip-tilt module (TTM) stabilizing the image of the star at the instrument aperture to ensure that the average image over a typical exposure time (of at least $10 \mathrm{sec}$ ) is stable with respect to the entrance aperture to better than $0.01^{\prime \prime} \mathrm{RMS}$ up to $\mathrm{H} \simeq 10$. This device works in conjunction with a nIR viewing camera (model Owl 640 from Raptor Photonics) coupled to a JH filter, looking at 


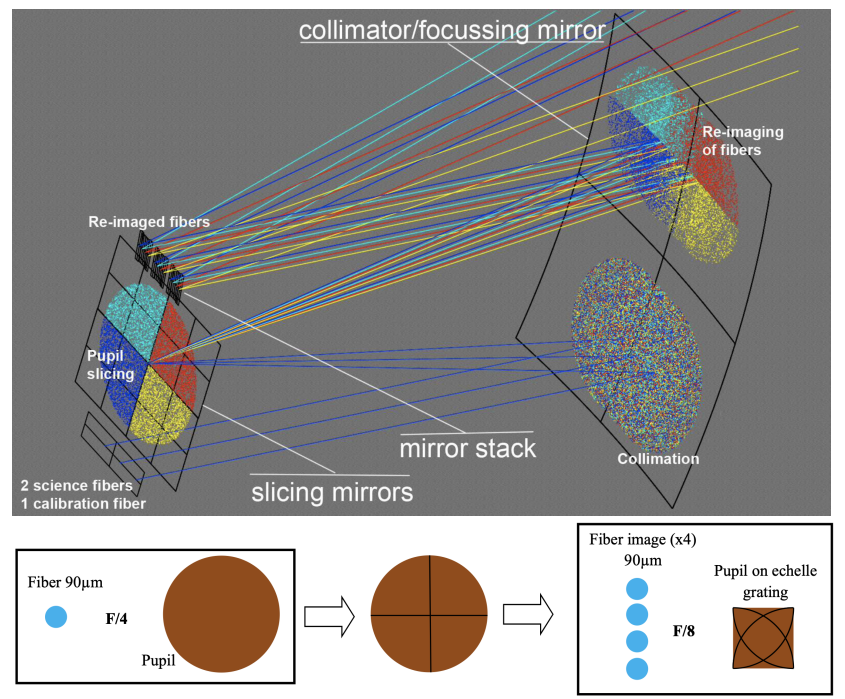

Figure 3. Optical layout of the SPIRou pupil slicer (top), with a schematic view of the pupil slicing principle for a single fibre (bottom)

the instrument entrance aperture and sending back information to the TTM at a frequency of up to $50 \mathrm{~Hz}$. The main goal of the TTM is to minimise shifts of the observed star with respect to the entrance aperture, and thereby the systematic RV errors that may result from these shifts when scrambling of the near-field image by the optical fibers is not perfect (see Sec. 3.2); at the same time, the TTM maximizes the stellar flux injected into the instrument. The algorithm implemented to measure the shift of the star with respect to the aperture (based on a least-squares Gaussian fit incorporating a low-reflectivity central circular aperture) and correct it through the TTM proved to be working well, both in lab tests and on-sky observations (Barrick et al. 2018). The final element of this module is a calibration wheel allowing one to inject light from the calibration unit (see Sec. 3.4) into the instrument, with linear polarization along various directions if need be.

The Cassegrain unit also includes the possibility of carrying an 'internal alignment verification' (IAV) by sending light backwards from a small $50 \mu \mathrm{m}$ fiber inserted in the fiber connector beside the main science fibers (see Sec. 3.2) into a second aperture $1 \mathrm{~mm}$ off the main instrument aperture in the field mirror; with a dedicated prism installed in the calibration wheel, one can monitor the position of the fiber image with respect to the second aperture using the viewing camera, and detect potential shifts (to a precision better than $1 \mu \mathrm{m}$ ) that may result from mechanical flexures or optical instabilities occurring between the main entrance aperture and the science fibers. Observed motions are of order $1 \mu \mathrm{m}$ RMS during observations across all telescope positions.

Altogether, the throughput of the Cassegrain unit is about $50 \%$ in the $\mathrm{K}$ band, falling down to $\simeq 30 \%$ in the $\mathrm{Y}$ band as the main result of ZnSe absorption from the rhombs. The Cassegrain structure was designed, assembled and tested in a dedicated clean room by the SPIRou team members at OMP/IRAP (Parès et al. 2012).

\subsection{The fiber link \& pupil slicer}

The fiber link features two 35-m long science fibers conveying the light from the twin orthogonally polarized beams coming out of the Cassegrain polarimeter to the spectrograph, plus a 10-m long reference fiber bringing light from the calibration unit to the spectrograph (see Sec. 3.4). All 3 fibers are circular fluoride fibers with a $90-\mu \mathrm{m}$ diameter, engineered by Le Verre Fluoré (LVF) in France from purified ZBLAN glass and ensuring a throughput of at least $90 \%$ over the entire spectral range of SPIRou. The fiber link also includes a 35-m long ZBLAN fiber (of diameter $300 \mu \mathrm{m}$ ) conveying the light of calibration lamps to the Cassegrain unit when needed, so that both science channels and the reference channel can be calibrated simultaneously in a homogeneous way.

On the Cassegrain side, the fiber link includes a fiber box that links the dual fiber head collecting light from the twin stellar images formed by the Wollaston prism, to a pair of single connectors, one for each of the two science fibers. Although increasing the number of fibers connections, this setup makes it far easier, safer and more repeatable to deconnect and re-connect the science fibers to the Cassegrain unit each time SPIRou is removed from the Cassegrain focus of the CFHT. On the spectrograph side, the fiber link features a triple hermetic feedthrough used to inject light from the three fibers within the cryostat (see Sec. 3.3). Following the feedthroughs, the fiber link includes a pupil slicer fed through three 1.4-m segments of $90-\mu$ m-diameter octagonal fiber (also engineered by LVF), each connected to a feedthrough. The pupil slicer per se consists of a doublepass collimator and a pupil-slicing mosaic of 4 flat mirrors located at the focus of the collimator and slicing the pupil into 4 equal $90^{\circ}$ sectors; the twelve individual images ( 4 images for each of the three fibers) formed after a second pass through the collimator are focused on a stack of twelve small flat mirrors ensuring that the pupils of all individual beams overlap into a square pupil once imaged onto the spectrograph grating (see Fig. 3). In addition of being extremely compact (only a few $\mathrm{cm}^{3}$ ), this device has the key advantage of ensuring a high throughput with no loss of resolving power, and of delivering sliced images with identical shapes and flux distributions (as opposed to more conventional image slicers for which slices have different shapes), a prime asset for precision velocimetry where image stability is critical.

Including absorption, FRD, connector and imaging losses, the fiber link provides an average throughput over the spectral range of $\simeq 60 \%$. The combination of the circular and octagonal fibers, although intended to ensure a scrambling of the near-field image between polarimeter output and spectrograph input of order 1000, was however found to be much less efficient, with a scrambling performance about $10 \times$ lower than expected; this degradation was recently showed to derive from excess stress in the way the pupil slicer holds the octagonal fibers. A hardware upgrade of the pupil slicer (with a stress-free mount of the octagonal fibers) is planned to improve near-field scrambling from the fiber link. Regarding thermal background, the contribution from the fibers is moderate, with only about $5 \mathrm{ph} \mathrm{s}^{-1} \AA^{-1}$ per fiber, whereas that from the hermetic feedthroughs, depending on the room temperature, is found to be about $25 \mathrm{ph} \mathrm{s}^{-1} \AA^{-1}$ at $2.35 \mu \mathrm{m}$ for a temperature of $15^{\circ} \mathrm{C}$. The 


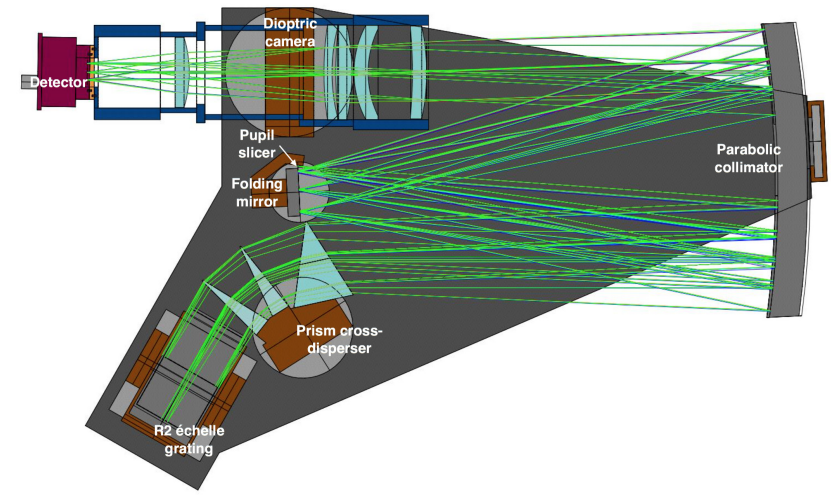

Figure 4. Optical layout of the SPIRou spectrograph

fiber link, designed, assembled and tested by the SPIRou team members at OMP/IRAP (Micheau et al. 2012, 2015, 2018).

\subsection{Cryogenic high-resolution spectrograph \& detector}

The SPIRou spectrograph is a bench-mounted highresolution échelle spectrograph in a dual-pupil optical layout similar to that of ESPaDOnS and HARPS (see Fig. 4), the main difference being that the prism-train cross-disperser is used in double pass ${ }^{3}$. Light is injected into the spectrograph by the pupil slicer fed with 3 separate fibers, each one entering the spectrograph through a dedicated hermetic feedthrough (see Sec. 3.2). The pupil slicer generates a telecentric $\mathrm{f} / 8$ beam and a slit-like image featuring a triple set of 4 circular slices of overall dimension $90 \times 1530 \mu \mathrm{m}^{2}$ (one set of 4 slices for each of the 3 incoming fibers). This $\mathrm{f} / 8$ beam first meets the main collimator (an off-axis parabola with 1200 $\mathrm{mm}$ focal length), projecting a $150-\mathrm{mm}$ square pupil onto the dispersing optical components, i.e., a double-pass tripleprism train cross-disperser (made of two $\mathrm{ZnSe}$ and one Infrasil prisms, of clear aperture $190 \times 206 \mathrm{~mm}$ ) and a R2 échelle grating (of clear aperture $154 \times 306 \mathrm{~mm}^{2}$, w/ $23.2 \mathrm{gr} / \mathrm{mm}$ ). Following a second pass on the off-axis parabola, the converging $\mathrm{f} / 8$ beam is reflected off a flat mirror near which an intermediate image of the full spectrum forms. This spectrum is then re-injected into a fully-dioptric 5-lens camera (500-mm focal length, 220-mm clear aperture) after a third pass on the main collimator, forming a converging $f / 3.33$ beam and the final scaled-down spectrum on a $4 \mathrm{k} \times 4 \mathrm{k}$ H4RG detector (with 15- $\mu \mathrm{m}$ square pixels) located at the focus of the camera.

With this design, the spectrograph is able to fit the entire nominal spectral range of SPIRou $(0.98-2.35 \mu \mathrm{m})$ on the H4RG detector (as 46 orders numbered \#78 to \#33) and even fit 2 additional orders on both sides (\#80 to \#31) to further extend the usable spectral domain $(0.95-2.50 \mu \mathrm{m})$ albeit with a small 2-nm gap between the two reddest orders. Each order is about 48-pixel wide, with the distance between consecutive orders varying from 53 to 96 pixels. The spot

\footnotetext{
3 The blue and red pupils on the grating are thus shifted by a few $\mathrm{mm}$ with respect to the pupil at mid wavelength, as visible on Fig 4.
}

diagrams (see Fig. 5) feature a full-width at half-maximum (FWHM) across the slit smaller than half a pixel in most orders and everywhere smaller than 0.65 pixel, ensuring that the spectrograph is diffraction limited over the full spectral domain. The spectral pixel size is equal to $2.28 \mathrm{~km} \mathrm{~s}^{-1}$ within $15 \%$ throughout the whole domain, staying virtually constant at the centre of all orders and varying across orders. The FWHM of the instrument response is dominated by the (non Gaussian) slicer profile $\left(\simeq 4 \mathrm{~km} \mathrm{~s}^{-1}\right)$, with minor contributions from the detector pixels $\left(1.8 \mathrm{~km} \mathrm{~s}^{-1}\right)$ and from the optical point-spread-function $\left(1.5 \mathrm{~km} \mathrm{~s}^{-1}\right)$; as a result of the non-Gaussian shape of the instrument profile, the resolving power as derived from narrow calibration lines $(\simeq 64 \mathrm{k})$ is predicted to be slightly smaller than that estimated from its broadening impact on the (wider) spectral lines of SPIRou stellar targets $(\simeq 70 \mathrm{k})$. Thanks to the tilted slit with respect to the detector pixels ensuring that the spectrum is sampled on a different pixel grid across the slit, it is possible to extract a spectrum with adequate sampling at full resolution, despite the detector pixel size (of $2.28 \mathrm{~km} \mathrm{~s}^{-1}$ ) being $6 \%$ larger than half the resolution element (of $4.29 \mathrm{~km} \mathrm{~s}^{-1}$ ).

The SPIRou H4RG detector is a science-grade device engineered by Teledyne Imaging Sensors (TIS, USA), then mounted on its Focal Plane Array and extensively tested by the SPIRou team members at Université de Montréal (UdeM, Canada, Artigau et al. 2018); at $\simeq 80 \mathrm{~K}$, it features a quantum efficiency ranging from 80 to $95 \%$ over the spectral domain, a cutoff wavelength of $\simeq 2.4 \mu \mathrm{m}$, a readout noise of $12 \mathrm{e}^{-}$in Correlated Double Sampling (CDS) readout mode, a median dark current of $0.007 \mathrm{e}^{-} / \mathrm{s}$, and an average crosstalk to neighbouring pixels of $0.8 \%$. The detector flatness is better than $10 \mu \mathrm{m}$ peak-to-valley $(\mathrm{PtV})$, with a RMS under $5 \mu \mathrm{m}$.

The camera, assembled at Université Laval in Quebec City (UL, Canada) and tested at cryogenic temperatures at UdeM, follows a concept similar to that of WIRCAM, CFHT's wide-field infrared camera (Thibault et al. 2003), with nylon pads holding the lenses to ensure minimal stress at operating temperatures. Its throughput ranges from $95 \%$ in the blue down to $90 \%$ in the $\mathrm{K}$ band (reflecting the reduced transmission of the fourth camera lens made of SFTM16). The parabola, engineered by SESO (France), features a protected silver coating ensuring a reflectivity of $98 \%$ over the full domain, with wave-front errors smaller than $\lambda / 2$ $\mathrm{PtV}$. The flat mirror and the prisms were engineered by Optical Surfaces (UK); the prism-train transmission peaks at $90 \%$ in the $\mathrm{K}$ band, falling down to $70 \%$ towards the blue as a result of the higher ZnSe absorption. Finally, the R2 grating, fabricated by Richardson Lab (USA), exhibits an average transmission of $70 \%$ throughout the domain, with wavefront errors smaller than $\lambda / 2 \mathrm{PtV}$. As a whole, the spectrograph exhibits a total throughput of $\simeq 50 \%$, falling down to $\simeq 30 \%$ on the blue side as a main result of ZnSe absorption. The spectrograph design was achieved by the SPIRou team members at Institut de Planétologie et d'Astrophysique de Grenoble (IPAG) in France, with contributions from team members at UL (Thibault et al. 2012).

The alignment of the optical components, achieved at room temperature, consists of 5 main steps. We start by installing four mechanical masks featuring pinholes at predefined locations, defining the reference path of the optical beam, and accurately positioned on the optical bench (in 

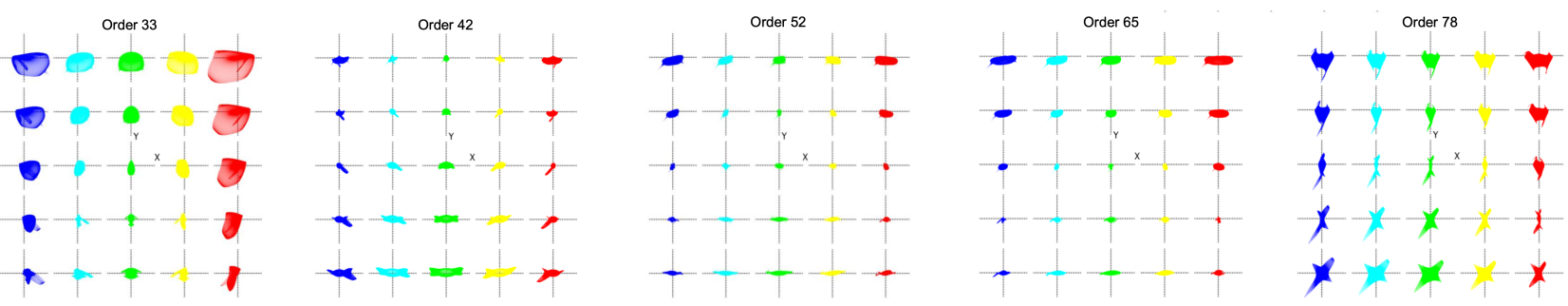

Figure 5. Spot diagrams of the SPIRou spectrograph across the full spectral range (for orders $33,42,52,65$ and 78 , corresponding to wavelengths $2.40,1.83,1.48,1.18$ and $0.99 \mu \mathrm{m}$ respectively), for 5 wavelengths across each order (X direction) and for 5 positions across the slit ( $\mathrm{Y}$ direction). The cross represents the size of one H4RG detector pixel $(15 \mu \mathrm{m})$.

front of the parabola and of the folding flat mirror) and on the parabola; with the dispersing elements (prisms and grating) replaced by a flat mirror located at the correct distance and angle, the parabola and the fold mirror are then tuned in position and orientation with respect to the reference masks on the optical bench until a collimated red laser beam passes through all pinholes. Using several paper targets, the dispersing components and the camera are then installed on the bench so that the spot from the collimated red laser and/or from a fibred nIR laser beam matches the expected position on each optical surface. Focusing the slicer and the folding mirror is achieved thanks to a large autocollimator (1-m focal length and 10-cm pupil), down to a precision of about $50 \mu \mathrm{m}$. The fourth step consists in tuning the detector focus and tilt by introducing calibrated shims in the detector mount until the proper setting is achieved, down to a precision of about $10 \mu \mathrm{m}$; this step is the most delicate one, requiring several cooling cycles to converge. In the last step, we move the parabola to its nominal 'cold position at room temperature', i.e., the position that it should have at room temperature to ensure perfect optical alignment and focus once the whole spectrograph reaches its operating temperature of $73.5 \mathrm{~K}$. Once all components on the spectrograph bench reached operating temperature, we use a Hartmann mask in front of the camera (alternatively hiding the top and bottom halves of the beam) to estimate the distance to the optimal focus for all regions of the detector; the detector is positioned with respect to the overall optimal focus to a precision better than $10 \mu \mathrm{m}$ (see Fig. 6). The spectrograph optical alignment was devised and optimized by the SPIRou team members at OMP/IRAP, to ensure a straightforward reassembly and realignment at the CFHT (Challita et al. 2018).

The whole spectrograph with its optical bench (of total weight $500 \mathrm{~kg}$ ) is enclosed in a cryogenic dewar (of external diameter $1.73 \mathrm{~m}$ and length $2.87 \mathrm{~m}$ ) and mounted on a table supported at three points by an hexapod system from an internal support frame at room temperature (for a total weight of 3.5 tons). The spectrograph and optical bench are evacuated (down to a typical pressure of $10^{-6} \mathrm{mbar}$ ) and cooled down to $73.5 \mathrm{~K}$ via two cryocoolers acting on a cold bus that spreads cold to the whole optical bench. The bench and spectrograph optics are enclosed in a multiple layer of active and passive thermal shields to further stabilize the temperature of all optical components to better than $1 \mathrm{mK}$. This high-level of thermal stability is required to ensure that the science channel does not drift in velocity with respect to

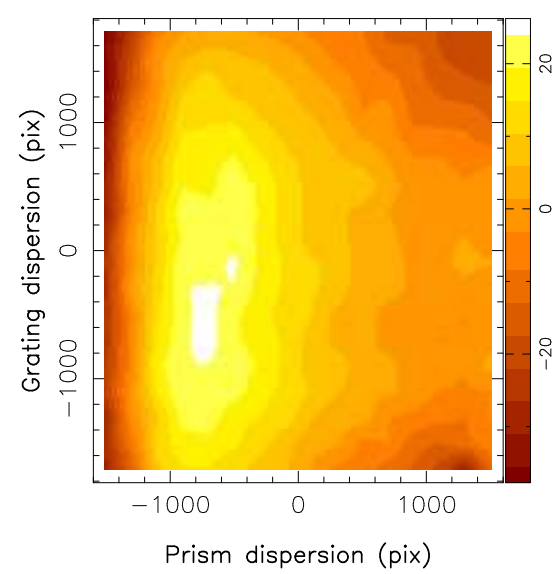

Figure 6. Distance to the optimal focus (in $\mu \mathrm{m}$ ) over a $3200 \times 3600$-pixel region covering most of the SPIRou spectrum (red is on the left and orders run vertically). The focus variations mostly reflect the longitudinal chromatism of the optical design, with a $9.5 \mu \mathrm{m}$ shift with respect to the optimal focus.

the reference channel by more than a fraction of a $\mathrm{ms}^{-1}$ in a timescale of one night.

The temperature of the optical bench is stabilized at three specific points (located below the grating, the camera and the parabola respectively) through 3 control loops equipped with Isotech MicroK-500 / Cernox CX-1080 sensors, ensuring a typical RMS stability of $0.2 \mathrm{mK}$ on timescales of $24 \mathrm{hr}$ (see Fig. 7 top panel); the temperature of the active shield is controlled with more standard components at a typical precision of a few mK RMS. The (uncontrolled) temperature of optical components and of various bench locations are constantly monitored every few seconds, and are all found to be stable to within $1 \mathrm{mK}$ RMS (see, e.g., Fig. 7 bottom panel showing the SPIRou grating temperature over a timescale of a week), demonstrating that the technical requirement on the thermal stability of the spectrograph is met. The typical cool-down time of the whole instrument from ambient temperature to $73.5 \mathrm{~K}$ is about $9 \mathrm{~d}$, whereas the additional time for all optical components to stabilize at a level of $1 \mathrm{mK}$ RMS is another $11 \mathrm{~d}$. With a warm up time of about $6 \mathrm{~d}$, the duration of a full thermal cycle is close to a month.

The design and construction of the cryogenic dewar were achieved by the SPIRou team members at the Hertzberg Institute of the National Research Council of Canada (NRC-H, Victoria, Canada, Reshetov et al. 2012), 


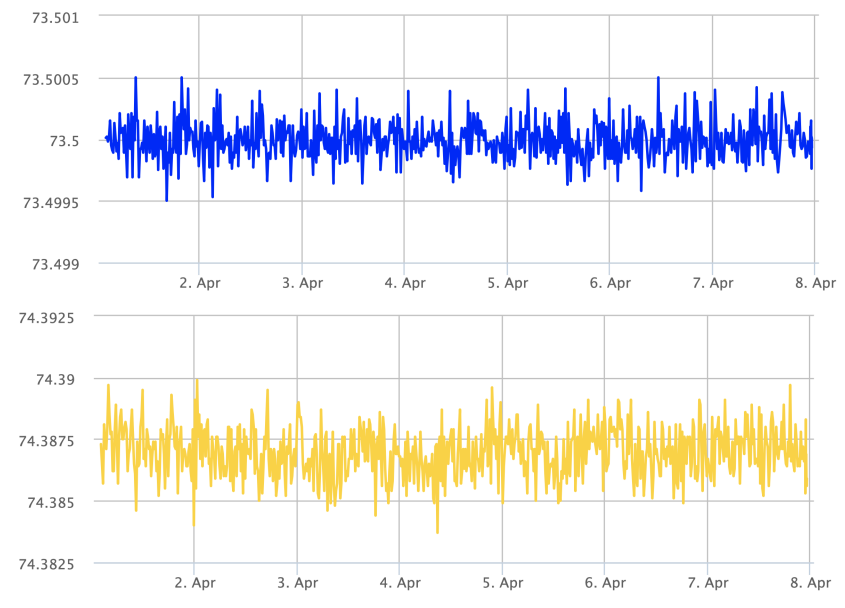

Figure 7. Temperature of one of the 3 control points of the SPIRou spectrograph bench (top panel) and of the (uncontrolled) SPIRou grating over a timescale of a week in early April 2020, respectively showing thermal stabilities of 0.2 and $1.0 \mathrm{mK}$ RMS over $24 \mathrm{hr}$ periods.

then delivered to OMP/IRAP where SPIRou was integrated and extensively tested in a clean room.

\subsection{Calibration \& RV reference unit}

The purpose of the calibration unit, located close to the spectrograph cryostat, is to provide light from the various lamps needed to accurately calibrate observed spectra. It includes:

- a halogen lamp (with a radiation temperature of $3000 \mathrm{~K}$ ) to collect the flat field (FF) exposures needed to locate orders on the detector and to correct for the spectral response of the instrument;

- a U/Ne hollow-cathode (HC) lamp featuring about $2 \mathrm{k}$ lines over the spectral domain, to secure a precise absolute pixel-to-wavelength calibration (a Th/ $\mathrm{Ar} \mathrm{HC}$ lamp is also available, with less lines over the domain and thus not used in operations);

- an evacuated $(\simeq 0.1 \mathrm{mbar})$ and temperature-stabilised ( $\simeq 1 \mathrm{mK}$ RMS on $24 \mathrm{hr}$ slots) Fabry-Perot (FP) etalon coupled to a halogen lamp, featuring about $15 \mathrm{k}$ lines across the spectral range, to track the detailed shape of the slit-like image that the pupil slicer generates at the spectrograph entrance, and to monitor spectrograph drifts during observations (with the FP spectrum on the reference fiber during on-sky observations) at a precision of a few $0.1 \mathrm{~m} \mathrm{~s}^{-1}$.

FP exposures are also used in conjunction with $\mathrm{HC}$ exposures to refine the wavelength solution over the whole spectral range, especially in the reddest orders where the $\mathrm{U} / \mathrm{Ne}$ lamp features very few lines (Hobson et al. 2020, submitted). During tests and daily calibrations, light from calibration lamps is also injected into the 2 science fibers via the calibration wheel of the Cassegrain unit, so that all 3 spectral channels can be calibrated together in a consistent and homogeneous way. Typical calibration and science frames are shown in Fig. 8. As the FP spectrum shifts by $1 \mathrm{~m} \mathrm{~s}^{-1}$ for internal pressure and temperature changes of 0.006 mbar and $3.1 \mathrm{mK}$ respectively, which can happen on a timescale of a few d, daily calibrations are essential so that all drifts can be accurately corrected for. The calibration unit was designed and integrated at Observatoire de Haute-Provence / Laboratoire d'Astrophysique de Marseille (OHP/LAM, France, Boisse et al. 2016; Perruchot et al. 2018), and the RV reference module was developed at Observatoire de Genève (OG, Switzerland, Wildi et al. 2012; Cersullo et al. 2017).

In addition to the standard calibration lamps integrated within the calibration unit and delivered with the instrument, SPIRou was recently equipped with a Laser Frequency Comb (LFC) engineered by Menlo Systems (MS, Germany). The SPIRou LFC features a repetition rate of $13.0 \mathrm{GHz}$ and an offset of $5.31 \mathrm{GHz}$, with lines covering a spectral range of 1.0-2.2 $\mu \mathrm{m}$, i.e., about $10 \%$ and $20 \%$ smaller in log scale than the nominal and overall SPIRou domains respectively (see Table 1). This limitation reflects the extreme challenge of manufacturing a tapered Photonic-Crystal Fiber (PCF) capable of generating the desired spectral broadening from the initial laser comb anchored at $1553 \mathrm{~nm}$. Up to now, MS succeeded in obtaining a hybrid LFC with a blue and a red arm, each arm feeding the multi-mode output LFC fiber with its own single mode fiber; whereas the red arm is equipped with an active flattening unit incorporating a spatial light modulator (SLM) supposed to trim all lines at a given intensity (within a few $\mathrm{dB}$ ), the blue arm only includes a filter-based passive flattening scheme (at a level of $13 \mathrm{dBs}$ ). As a result of time-variable fringing patterns in the SLM, line intensities in the red arm of the LFC are found to vary by up to $20 \mathrm{~dB}$ over the red range (and up to $10 \mathrm{~dB}$ on regions of only a few lines). Work is ongoing at MS to manufacture a new tapered PCF that can generate a spectral broadening matching the full range of SPIRou in one shot, and in upgrading the flattening unit and its SLM so that the LFC can achieve nominal performance in terms of homogeneity and stability of line intensities. An example LFC frame is shown in Fig. 8 (bottom left panel), where the residual fringe pattern is clearly visible. Once upgraded, the LFC will also be ideal for monitoring the SPIRou instrumental profile over time.

\subsection{Installation \& operation at the CFHT}

SPIRou was first integrated and tested at OMP/IRAP until 2017 December, then shipped to the CFHT. The spectrograph was installed in the Coudé room at the 3rd floor of the telescope building in 2018 February, whereas the spectrograph control electronics, the compressors and the calibration unit were installed in the adjacent room where the Coudé pipe (coming from the 5 th floor) emerges. The Cassegrain unit is mounted at the $\mathrm{f} / 8$ Cassegrain focus of the telescope, and connected to the spectrograph and calibration unit by the fiber link rooted through the Coudé pipe (with a $15^{\circ}$ Ctemperature difference between the two). SPIRou underwent extensive on-site testing throughout 2018, both in the lab and on the sky, until successfully passing the final acceptance review in 2019 January.

Instrument control was designed to operate in a CFHTlike environment, with a master process (the Director) controlling a number of slave processes (Agents), each in charge of a SPIRou subsystem (such as the Cassegrain unit, the spectrograph temperature control, the calibration unit). The most challenging aspects were the control of the TTM on the 

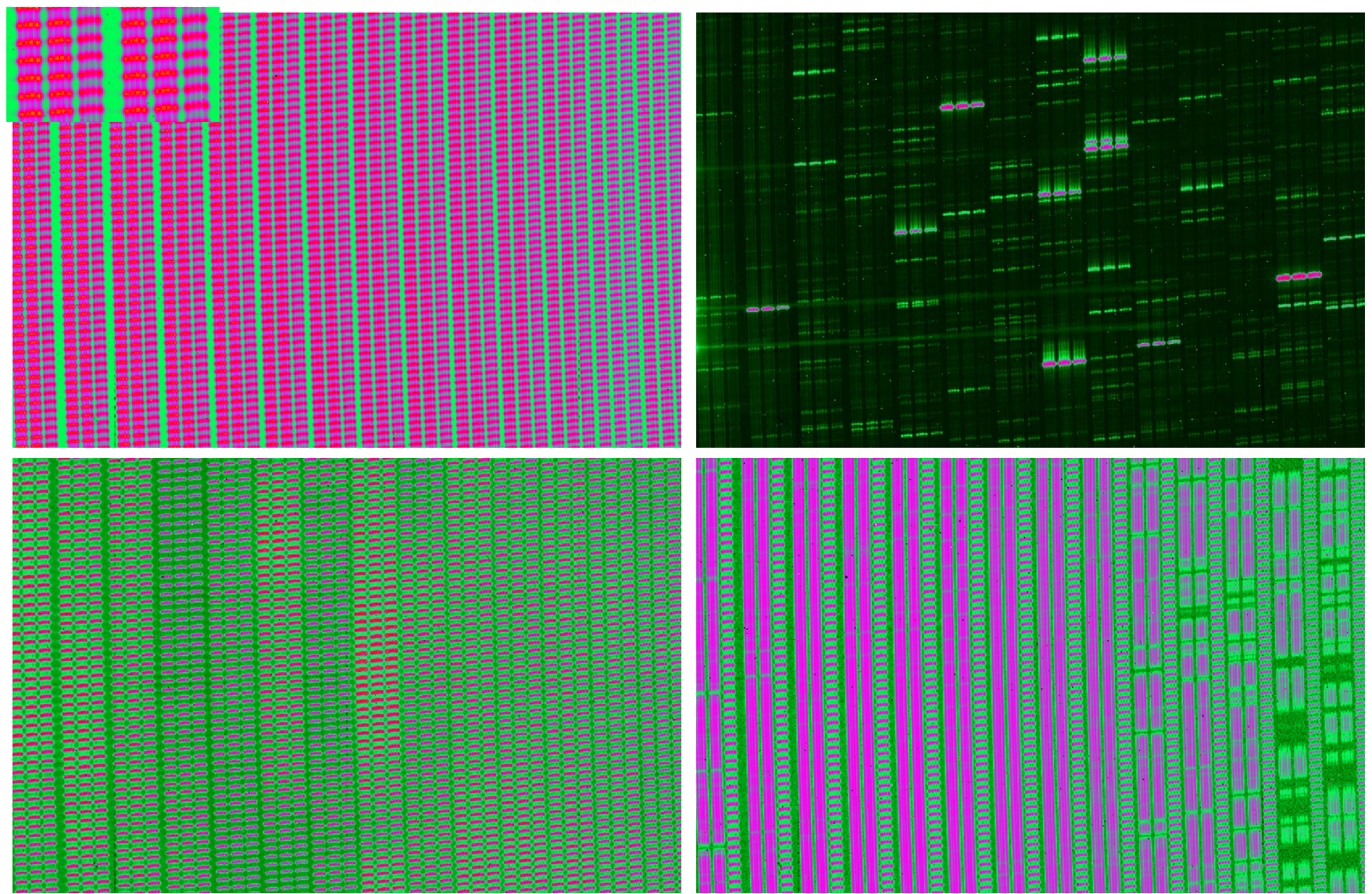

Figure 8. Examples of SPIRou calibration and science frames collected on 2020 March 11, in the case of the FP (top left), HC (top right), LFC (bottom left) and science+FP exposures (for the active M dwarf AD Leo). The image only shows a portion at the centre of the detector, with 14 (out of the 50) recorded orders (running vertically, from order \#43 on the left to \#56 on the right, corresponding to mid wavelengths of 1787 to $1373 \mathrm{~nm}$ ). Each order displays a restricted spectral interval about flux maximum, and features 3 spectra, the left and middle ones corresponding to the science channels, and the right one associated with the reference channel. In the FP, HC, and LFC frames, all 3 channels feature calibration spectra, whereas the science frame exhibits the spectrum of AD Leo (with both stellar and telluric absorption lines clearly visible) accompanied by a FP spectrum on the reference channel. The insert in the top left image is a zoom on the 3 channels of two consecutive orders, showing the quadruple-slice pattern of each channel.

Cassegrain side (to ensure optimal behaviour up to $50 \mathrm{~Hz}$ ), and the temperature control on the spectrograph side (to achieve the best possible thermal stability of the optical components). More details can be found in Barrick et al. (2012) and in Barrick et al. (2018). All passive and active elements / probes are constantly monitored, with data on timescales ranging from $1 \mathrm{hr}$ to $1 \mathrm{wk}$ being accessible from the web on a 'status server'.

Agitators were added to all fiber cables linking the different instrument modules (calibration and Cassegrain units, spectrograph, RV reference, LFC), to ensure optimal scrambling of the modal noise, that can impact observations in the nIR, especially in the reddest orders. Significant progress was achieved since SPIRou was installed at the CFHT, though there is still room for improvements, in particular with the LFC whose nearly monochromatic spectral lines are extremely sensitive to modal noise.

Shortly after the installation of SPIRou, the CFHT suffered a $7^{\text {th }}$ magnitude earthquake, following which SPIRou became more sensitive to vibrations, e.g., induced by earthquakes or even by the activation of the telescope hydraulics, and causing the RV in both science and reference channels to jump by tens of $\mathrm{m} \mathrm{s}^{-1}$. Fortunately, relative RVs between the science and reference channel are unaffected by these events. We suspect that this problem reflects a crack in one of the glue joints that bond the optical components to their mechanical supports, most likely for one of the prisms given the preferred direction of the observed spectral shifts. SPIRou also suffered two breakdowns of its cooling system up to now (in 2019 August and 2019 December), each time due to a failure of a cold-head motor whose fix required to warm-up the whole instrument; now equipped with more robust components, and with the planned maintenance of the whole cooling system every second year, cold heads should no longer be a source of issues in the future.

Considerable efforts were also invested into cooling down the Coudé room where SPIRou is installed. With an average room temperature that jumped from about 12 to $20^{\circ}$ Cfollowing the installation of SPIRou, the thermal background caused by the hermetic feedthroughs reached a high point of about $40 \mathrm{ph} \mathrm{s}^{-1} \AA^{-1}$ at $2.35 \mu \mathrm{m}$. Heat extraction was implemented (especially in the adjacent room hosting the spectrograph compressors) to bring the Coudé room back to a temperature of about $15^{\circ} \mathrm{C}$, and further action is under- 
going to further decrease it to about $10^{\circ} \mathrm{C}$ (e.g., Look et al. 2018). Active cooling is also being implemented on the hermetic feedthroughs to attempt bringing their temperature down to $5^{\circ} \mathrm{C}$.

A hardware upgrade is planned for early 2021, aiming at reducing SPIRou's sensitivity to vibrations, at changing the pupil slicer (now including a stress-free mount of the octagonal fibers) to enhance the near-field scrambling efficiency, and at improving the cooling performance on the hermetic feedthroughs in order to further decrease their (currently dominant) contribution to the thermal background (see Secs. 3.2 and 4)

Science observations, including the SLS, started in 2019 February at a rate of about 130 nights per year (mostly during bright time), with SPIRou being operated in Queue Service Observing (QSO) mode from the start. On-sky science SPIRou data either consist of individual exposures collected in a fixed rhomb configuration, or of (circular or linear, i.e., Stokes $V, Q$ or $U$ ) polarization sequences of 4 subexposures in pre-defined rhomb orientations. In addition to the science programmes being carried out, SPIRou nightly collects exposures on a few telluric and RV standard stars, as well as on the sky. SPIRou spectra of calibration lamps are also recorded twice a day, at the beginning and at the end of each SPIRou observing night. An average SPIRou night is found to last $7 \mathrm{hr}$ of science observing time (and read-out), once pointing overheads, night calibration time and losses to weather are discounted.

\subsection{Data reduction pipeline}

The SPIRou reduction pipeline, called APERO (standing for A PipelinE to Reduce Observations, Cook et al. 2020, in prep), works in several steps. The first one consists in analyzing calibration files collected at the beginning and end of any given observing night, to derive the geometrical elements (shape of orders from FF frames, shape of slit from FP frames, position of lines in $\mathrm{HC}$ and $\mathrm{FP}$ frames) thanks to which all frames from this observing night can be straightened into a reference coordinate system associated with a set of reference calibration files. In a second step, stellar spectra on the science channel (and their reference FP spectra in the reference channel whenever relevant) are extracted from each straightened science frame using optimal extraction (Horne 1986), and wavelength calibrated using a master pixel-to-wavelength conversion formula also derived from the set of reference calibrations (using lines from both $\mathrm{HC}$ and FP frames, see Sec. 3.4, as in, e.g., Hobson et al. 2020, submitted). Finally, stellar spectra are corrected from telluric lines using a PCA approach applied to a data base of SPIRou spectra of telluric standards collected in a wide range of atmospheric conditions (with several such standards observed every single SPIRou night, further expanding the data base, Artigau et al. 2014, Artigau et al. 2020, in prep); spectra are also automatically corrected for the Barycentric Earth Radial Velocity (BERV).

Processed (telluric uncorrected and corrected) spectra are saved in several formats, with both 2D FITS files containing the extracted unnormalized spectra of all orders (e2ds files, which can be flattened later-on using a similar s2d file from a FF frame), and 1D FITS files containing flattened spectra with all orders merged together and re- binned on a wavelength grid featuring a constant velocity (or wavelength) step. At the CFHT, an automatic trigger ensures that each new recorded SPIRou frame, either calibration or science ones, are processed by APERO as they are being collected; in a second step, full reprocessing of all data from the night is carried out to obtain the final reduction products. Whenever individual exposures are part of a polarization sequence, APERO also computes a polarization spectrum from the extracted spectra of individual exposures (e.g., Moutou et al. 2020; Martioli et al. 2020).

In the case of polarization sequences (i.e., most SLS data), consisting of series of 4 consecutive sub-exposures collected in different positions of the Fresnel rhombs (to remove systematics to first order, Donati et al. 1997), APERO produces the same kind of data output, along with the corresponding polarization spectra (for the selected polarization state / Stokes parameter) and a null polarization spectrum (with the 4 subexposures processed in a different way so that polarization signatures cancel out, Donati et al. 1997).

In addition to the extracted SPIRou spectra, APERO generates several by-products, e.g., the cross-correlation function $(\mathrm{CCF})$ of the spectrum with a line mask matching best the spectral type of the observed target, from which preliminary RV estimates are obtained. (In the near future, APERO will incorporate a larger set of line masks than the current limited sample of 4). Zeeman signatures will also be derived using Least-Squares Deconvolution (LSD Donati et al. 1997) as a by product in the case of polarization sequences. Additional work may be needed from the PIs, in particular regarding telluric correction, to further optimize the derivation of precise RVs and accurate Zeeman signatures.

Currently in version 0.6.100, APERO is available from GitHub, and is still undergoing regular updates to improve reduction performances, in particular regarding telluric correction and RV precision (Artigau et al. 2012, Cook et al. 2020 , in prep). Alternative pipelines are also being developed in parallel (one being based on the ESPaDOnS pipeline Libre-ESpRIT, Donati et al. 1997), to compare with APERO results and ultimately ensure that reduction recipes are optimized so that SPIRou data can be exploited at their best.

\subsection{The SPIRou project team}

Including design, assembly, integration, tests and installation at the CFHT, SPIRou took about a decade to come to life, with the initial CFHT decision to build SPIRou dating from 2010, the Final Design Review occurring in late 2014, the pre-shipping and final acceptance reviews passed in 2017 December and 2019 January respectively. Over this decade, the SPIRou project team involved about 50 engineers and scientists from 10 institutes around the world, managed by OMP/IRAP hosting the Project Manager (PM), the Principal Investigator (PI), the deputy PM and the System Engineer. IPAG hosted the SPIRou Project Scientist (PS), UdeM provided the deputy PI and the deputy PS whereas CFHT allocated the Observatory Scientists. In each of the main partner sites (IRAP/OMP, OHP-LAM and IPAG in France; CFHT in Hawaii; NRC-H, UdeM and UL in Canada; OG in Switzerland), a local PM was in charge of the handled subsystem; additional contributions were provided by the Institute of Astronomy \& Astrophysics of Academia Sinica 
Table 1. Summary of the main characteristics and performances of SPIRou

\begin{tabular}{|c|c|}
\hline Nominal spectral range in a single exposure (w/ no gaps) & $0.98-2.35 \mu \mathrm{m}$ in 46 orders $(\# 78$ to $\# 33)$ \\
\hline Overall spectral range (w/ a 2-nm gap between 2.4371 and $2.4391 \mu \mathrm{m}$ ) & $0.95-2.50 \mu \mathrm{m}$ in 50 orders $(\# 80$ to \#31) \\
\hline Spectral resolving power estimated from calibration lines & $64 \pm 1 \mathrm{k}$ \\
\hline Spectral resolving power estimated from stellar spectra & $70 \pm 3 \mathrm{k}$ \\
\hline Velocity bin size of detector pixel & $2.28 \mathrm{~km} \mathrm{~s}^{-1}$ \\
\hline On-sky diameter of circular instrument aperture & $1.29^{\prime \prime}$ \\
\hline Tip-tilt / guiding precision on instrument aperture (RMS) & $0.01^{\prime \prime}$ up to $\mathrm{H} \simeq 10$ \\
\hline Throughput & $3.5 \% / 7 \% / 10 \% / 12 \%$ in YJHK bands \\
\hline SNR performances & peak $\mathrm{S} / \mathrm{N} \simeq 110$ per pixel at $\mathrm{H} \simeq 8$ in $300 \mathrm{~s}$ for a $\mathrm{M} 4$ dwarf \\
\hline Polarimetric performances & circular \& linear, sensitivity 10 ppm, crosstalk $\simeq 1 \%$ \\
\hline Radial-velocity stability (RMS on standard stars) & $\simeq 2 \mathrm{~m} \mathrm{~s}^{-1}$ on a timescale of a few weeks, goal $1 \mathrm{~m} \mathrm{~s}^{-1}$ \\
\hline Equivalent $\mathrm{H}$ magnitude of thermal background at $2.35 \mu \mathrm{m}$ & 8.6, goal 9.5 \\
\hline
\end{tabular}
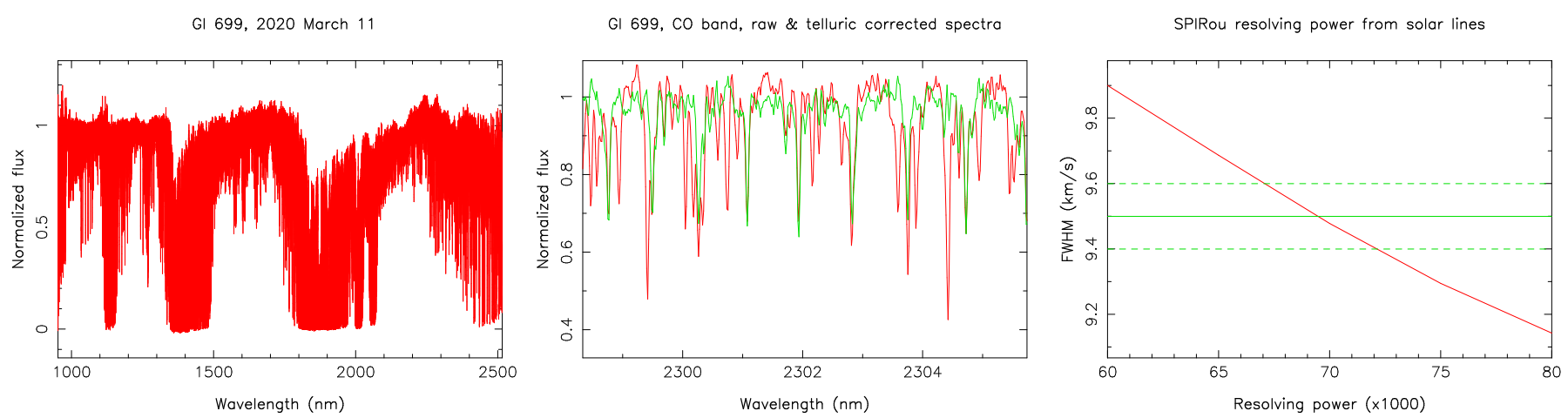

Figure 9. Left: SPIRou spectrum of the M dwarf Gl 699 over the full spectral domain. Middle: Small spectral region of Gl 699 in the ${ }^{12} \mathrm{CO}(2,0)$ band, before (red) and after (green) telluric correction. Right: FWHM of the LSD profiles of solar lines for various resolving powers. The green lines indicate the value and error bars estimated from SPIRou data, whereas the red line traces the prediction computed from a very high-resolution nIR spectrum of the Sun.

(ASIAA, Taiwan), the Laboratório Nacional de Astrofísica (LNA, Brazil) and the Instituto de Astrofísica e Ciências do Espaço (IA, Portugal).

\section{IN-LAB AND ON-SKY PERFORMANCES}

In this section, we summarize the performances of SPIRou regarding the main design specifications, which we recall in Table 1.

\subsection{Spectral domain \& resolving power}

Ranging all the way from 0.95 to $2.50 \mu \mathrm{m}$ with only a small gap (between 2.4371 and $2.4391 \mu \mathrm{m}$ ), the overall spectral domain of SPIRou is $\simeq 10 \%$ larger (in log scale) than the nominal window on which the optical design was optimized. We show in Fig. 9 (left panel) a SPIRou spectrum of Gl 699 secured on 2020 March 11, where the Y, J, H and K bands lie between regions featuring strong telluric absorption (centred at $1.14,1.40$ and $1.90 \mu \mathrm{m}$ ).

The resolving power of SPIRou as measured from $\mathrm{U}$ lines in $\mathrm{HC}$ frames is equal to $64 \pm 1 \mathrm{k}$, in agreement with design predictions. Reducing the pupil in front of the spectrograph camera to a $75 \mathrm{~mm}$ circular aperture (with the help of the Hartmann mask) degrades the resolution (by about $10 \%$ in the red), demonstrating that the spectrograph is in- deed diffraction limited and that optical aberrations have a small impact on the FWHM of the instrument profile.

Estimating the resolving power of SPIRou from the Gaussian broadening impact of the instrument on stellar spectra requires one to have in hand a very high-resolution reference spectrum of the star to be observed with SPIRou, so that the profiles of spectral lines can be compared. As the most obvious reference for this test is the Sun for which very high-resolution nIR spectra are available in the literature (e.g., Reiners et al. 2016), we observed the spectrum of the Sun (reflected off the Moon) with SPIRou, computed a LSD profile of all lines showing no (or very small) telluric contamination, and measured its FWHM on a velocity interval of $\pm 16 \mathrm{~km} \mathrm{~s}^{-1}$ ( \pm 7 pix) about the line center; repeating the exact same operation on the reference spectrum of the Sun smeared at different resolving powers (ranging from $60 \mathrm{k}$ to $80 \mathrm{k}$ ), we find that the Gaussian broadening impact of SPIRou corresponds to a resolving power of $70 \pm 3 \mathrm{k}$, in agreement with design predictions (see Fig. 9 right panel).

\subsection{Throughput \& thermal background}

The overall throughput of SPIRou (injection and atmospheric losses excluded) peaks slightly above $\simeq 10 \%$ in the $\mathrm{K}$ band (see Fig 10 left panel), dropping progressively towards the blue side of the domain as a result of ZnSe absorption (from the polarimeter rhombs and the spectrograph prisms), and falling sharply towards the red mostly due to the detec- 
SPIRou throughput

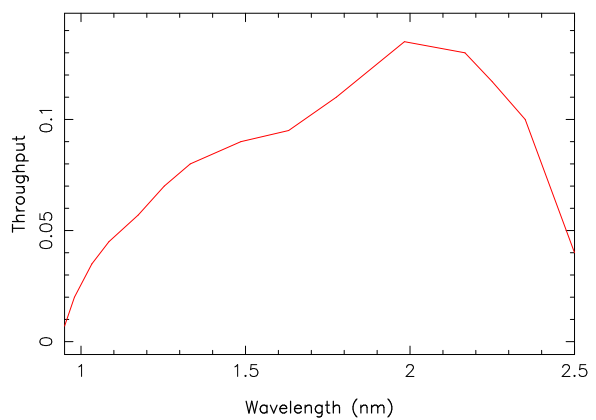

Gl $699-2020$ June $30-T \exp =61 \mathrm{~s}$

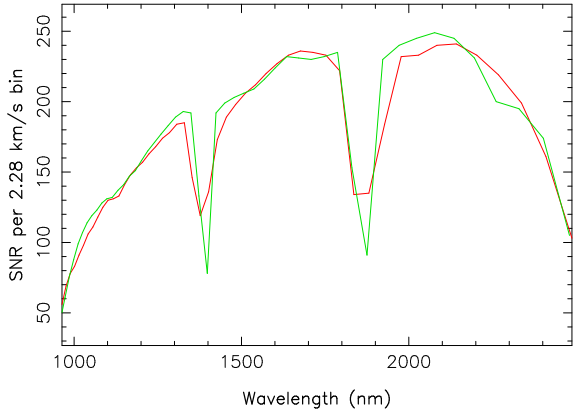

GJ $1151-2020$ June $30-$ Texp=301s

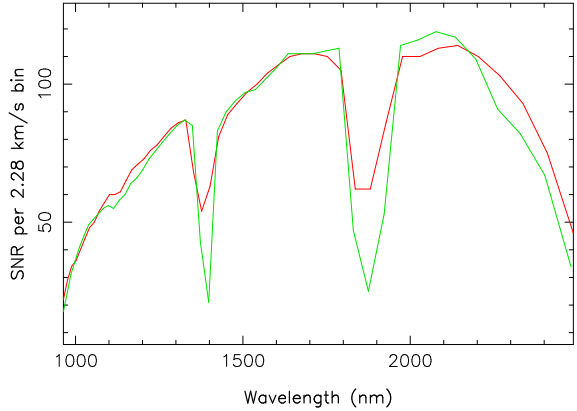

Figure 10. SPIRou overall throughput (injection and atmospheric losses excluded, left panel) and SNR curves for $61 \mathrm{~s}$ and $301 \mathrm{~s}$ exposures on the M dwarfs $\mathrm{Gl} 699(\mathrm{H}=4.83)$ and GJ $1151(\mathrm{H}=7.95)$ typical of SLS-PS targets (middle and right panels respectively) over the whole spectral domain (except in regions where telluric lines are too dense for the stellar continuum to show up, i.e., around 1.40 and $1.85 \mu \mathrm{m}$, see Fig. 9 left panel). The green lines are the observations (secured on 2020 June 30 in good weather conditions) and the red lines the ETC predictions (not taking into account thermal background).

Relative RV shift between science and reference channels

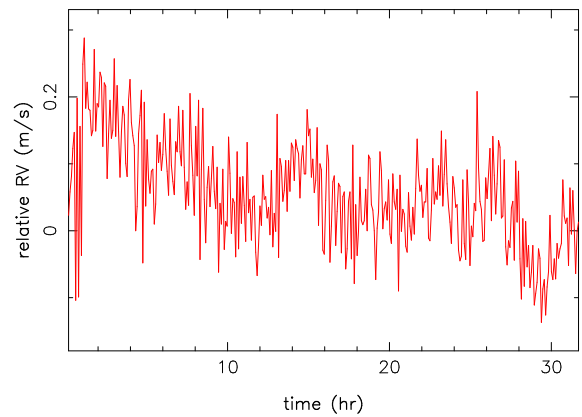

GI $514-2019$ May 14

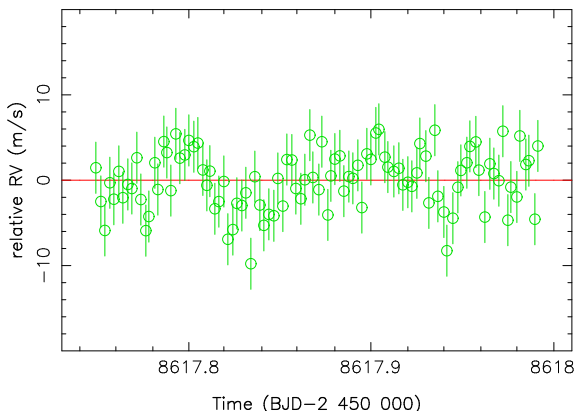

Gl $699-2019$ Oct $02-14$

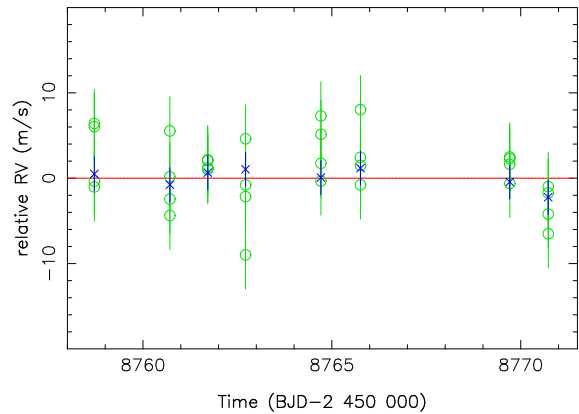

Figure 11. Monitoring the relative RV between the science and reference channels, through a $32 \mathrm{hr}$ test run with the FP RV reference injected in both channels (left panel), a 6-hr science run on the M2 dwarf Gl 514 (100× 180-s exposures with fixed rhomb configuration, middle panel) and a $13 \mathrm{n}$ run on the M4 dwarf Gl 699 (8 visits of $4 \times 61$-s polarimetric sequences, right panel). Green circles and blue crosses respectively indicate RVs on individual stellar exposures and on polarimetric sequences, with associated error bars set to 4 and $2 \mathrm{~ms}^{-1}$. The corresponding dispersions on relative RVs are respectively equal to $0.07 \mathrm{~m} \mathrm{~s}^{-1}, 3.4 \mathrm{~m} \mathrm{~s}^{-1}$ and $1.0 \mathrm{~m} \mathrm{~s}^{-1}$ (considering polarimetric sequences in the case of Gl 699), while the estimated photon noises are $0.03 \mathrm{~m} \mathrm{~s}^{-1}, 2 \mathrm{~m} \mathrm{~s}^{-1}$ and $0.5 \mathrm{~m} \mathrm{~s}^{-1}$.

tor cutoff (at $2.4 \mu \mathrm{m}$ ). This leads to predicted SNR curves with the SPIRou Exposure Time Calculator like those shown in Fig 10 (middle and right panels) in the case of the mid to late M dwarfs Gl 699 and GJ 1151 for exposure times of 61 and $301 \mathrm{~s}$ respectively, in reasonable agreement (where telluric lines are not too strong and dense) with observations secured in good weather conditions. Injection losses depend mostly on seeing, increasing from $10 \%$ to $50 \%$ when seeing degrades from 0.6 to $1.2^{\prime \prime}$.

Thermal background sums up to a total of $90 \mathrm{ph} \mathrm{s}^{-1} \AA^{-1}$ at $2.35 \mu \mathrm{m}$ for the SPIRou science channel, when adding the contributions of the polarimeter, fibers and hermetic feedthroughs (for polarimeter and feedthrough temperatures of about 2 and $15^{\circ} \mathrm{C}$ respectively). This corresponds to the stellar flux of a mid-M dwarf of magnitude $\mathrm{H} \simeq 8.6$ at $2.35 \mu \mathrm{m}$. The impact of the thermal background on SNR is negligible for bright stars like Gl 699, but starts to show up in the reddest orders for stars with $\mathrm{H} \simeq 8$ (like GJ 1151, see Fig. 10 right panel where the achieved SNR is below the prediction redder than $2.25 \mu \mathrm{m}$, i.e., for orders \#34 to \#31) and becomes dominant for targets fainter than $\mathrm{H} \simeq 9$. By cooling down the hermetic feedthroughs down to $\simeq 5^{\circ} \mathrm{C}$ (see Sec. 3.5), we hope to improve the situation and lower the thermal background to a stellar equivalent $\mathrm{H}$ magnitude of about 9.5.

\subsection{RV precision \& telluric correction}

$\mathrm{RV}$ precision is estimated by measuring the relative RVs between the science and reference channel and computing the dispersion on these measurements. Various contributors participate to the RV precision budget, in particular the instrument intrinsic stability, the light injection stability (of both near and far fields) and the pollution from telluric lines (that are variable with time in both position and strength with respect to stellar lines).

The first term of this budget can be easily estimated with in-lab tests, e.g., by injecting the light from our RVreference FP etalon in both the science and the reference channels simultaneously. The left panel of Fig. 11 shows the result of such a test (routinely done at the CFHT to monitor the instrument behaviour), where SPIRou exhibited a RMS stability of $0.07 \mathrm{~m} \mathrm{~s}^{-1}$ in relative RV over a timescale of $32 \mathrm{hr}$. When rotating the rhombs, this budget used to increase to $0.40 \mathrm{~m} \mathrm{~s}^{-1}$ as a result of the residual beam deviation (of a few ", i.e., a few $\mu \mathrm{m}$ at the fiber level). With the latest 
rhomb combination mounted in SPIRou in late 2020 June, no more RV jitter is detected so that the overall instrumental contribution to the RV budget is $0.1-0.2 \mathrm{~m} \mathrm{~s}^{-1}$ RMS.

Similar experiments were also carried out with the SPIRou LFC used as a RV reference. A relative RV precision of $\simeq 0.2 \mathrm{~m} \mathrm{~s}^{-1} \mathrm{RMS}$ was achieved up to now, which is encouraging, but still below the extreme RV precision (of a few $\mathrm{cm} \mathrm{s}^{-1}$ RMS) a LFC has the potential to deliver (e.g., Probst et al. 2020). This limitation reflects various technical problems encountered so far. For instance, the RV jitter in red orders is often 2 to $3 \times$ larger than that in blue orders, likely indicating the presence of residual modal noise in the fibers despite the implementation of fiber agitators and scrambling devices at various points along the link between the LFC and the SPIRou calibration unit; modal noise is indeed notoriously difficult to filter from LFC spectra, prone to this problem as a result of the monochromatic nature of individual lines. Moreover, flux variations accompanied by time-dependent RV shifts between the red and blue LFC orders are observed at times, which may be caused by injection fluctuations and differences between the two arms in the current hybrid LFC setup (see Sec. 3.4); a maintenance visit from MS is needed at this stage to further improve the overall LFC stability so that it can progressively replace the $\mathrm{HC}$ and become the ultimate absolute RV reference it was designed to be.

The second term of the RV precision budget (i.e., impact of light injection) is more tricky to evaluate through lab tests, requiring a device called 'artificial star' (AS) to inject light within the instrument in a way that mimics the behaviour of real stars, flickering under the effect of atmospheric turbulence and variable weather. Whereas near-field perturbations can be simulated with the AS, and mostly corrected for by the TTM to an accuracy of $0.01^{\prime \prime}$ RMS (i.e., $1.4 \mu \mathrm{m}$ RMS at the level of the instrument aperture, e.g., Barrick et al. 2018), far-field perturbations are much more tricky to generate in a realistic way, and can only be estimated on the sky with real stars, along with the impact of spectral pollution from telluric lines (the third main term in the RV precision budget).

The middle panel of Fig. 11 shows one such experiment carried out with SPIRou in 2019 May 14, where the bright inactive M2 dwarf Gl 514 was observed continuously for $6 \mathrm{hr}$ (100× 180-s exposures with fixed rhomb configuration) to study the relative RV jitter of individual exposures (with SNRs per pixel in H ranging from 220 to 280) that results from weather fluctuations and atmospheric variability. The inferred relative RV precision is $3.4 \mathrm{~m} \mathrm{~s}^{-1} \mathrm{RMS}$ over the $6 \mathrm{hr}$ run, and improves to $\simeq 2.5 \mathrm{~m} \mathrm{~s}^{-1}$ on shorter timescales, with a median dispersion of $2.6 \mathrm{~m} \mathrm{~s}^{-1}$ on groups of 4 consecutive exposures, typical to individual exposures of polarimetric sequences and $\simeq 1.5 \times$ larger than the expected photon-noise per exposure (1.5-2.0 $\mathrm{m} \mathrm{s}^{-1}$ depending on SNR). We note the presence of correlated noise (e.g., around JD=2 458617.8), whose origin is unclear as no correlation shows up with guiding parameters or atmospheric conditions, as would be expected if induced by residual near- and far-field perturbations not fully filtered out by the fiber link, or imperfect telluric correction (see, e.g., Fig. 9 middle panel).

Over the longer term, observations of inactive M dwarfs, such as Gl 699, are regularly being carried out as polarimetric sequences of 4 individual exposures, like in the 13-night
SPIRou run of 2019 October during which 8 sequences of $4 \times 61$-s individual exposures (each with a median SNR per pixel of 180 in $\mathrm{H}$ ) were collected. The relative RV dispersion of these observations is $1.0 \mathrm{~m} \mathrm{~s}^{-1} \mathrm{RMS}$ for polarimetric sequences (see Fig. 11 right panel), whereas the dispersion of RV measurements from individual exposures within polarimetric sequences ranges between 0.5 and $4.8 \mathrm{~m} \mathrm{~s}^{-1}$ (with a median value of $3.1 \mathrm{~m} \mathrm{~s}^{-1}$ ); if weighting the relative $\mathrm{RVs}$ by the inverse variance within each polarization sequence, the RV precision on this series of data points reaches $0.7 \mathrm{~m} \mathrm{~s}^{-1}$. We note in particular that no systematic pattern is observed between RVs of individual exposures within polarimetric sequences, in agreement with lab tests indicating that rhomb rotation is not degrading RV precision beyond a few $0.1 \mathrm{~m} \mathrm{~s}^{-1} \mathrm{RMS}$.

Altogether, these observations indicate that the relative RV precision of SPIRou is currently of order $2 \mathrm{~m} \mathrm{~s}^{-1}$ on a timescale of a few weeks for spectra of mid-M dwarfs with SNRs of $\simeq 200$, and is expected to regularly improve as upgrades are implemented, both on hardware (e.g., scrambling of the octagonal fibers feeding the pupil slicer, throughput and RV impact of rhombs) and on software (e.g., telluric correction). In particular, detector persistence is found to be an issue on the faintest targets $(\mathrm{H}>10)$, with exposures taken earlier in the night (especially on bright stars) having in some cases a significant impact on the RV estimate; similarly, FP spectra recorded in the reference channel may affect the spectra of faint targets in the science channels as a result of pixel crosstalk. Whereas the latter effect is now reliably corrected for within APERO, the former is currently under investigation so that the achievable RV precision is better quantified for the faintest targets.

\subsection{Polarimetric sensitivity \& crosstalk}

By observing the integrated Sun through its reflection on the Moon and carrying out a $4 \times 60$-s circular polarization sequence, a ultra-high SNR spectrum $(\mathrm{SNR} \simeq 2000$ per pixel in $\mathrm{H}$ ) was secured, from which Stokes $I$ and $V$ LSD profiles were computed (following telluric correction), yielding a noise level as low as $10 \mathrm{ppm}$ per pixel in the Stokes $V$ LSD profile (see Fig. 12 left panel). As in Donati et al. (1997), this test, showing that no spurious polarization is detected in conjunction with the narrow spectral lines of the Sun, demonstrates that SPIRou has the potential to reliably recover Zeeman signatures with a polarimetric sensitivity of at least $10 \mathrm{ppm}$, provided that enough photons are collected from the observed star. In the case of the Sun, no magnetic field is detected down to a precision of $0.2 \mathrm{G}$.

SPIRou is also able to detect polarized Zeeman signatures in the line profiles of known magnetic stars, such as the active $\mathrm{M}$ dwarf $\mathrm{AD}$ Leo and the chemically-peculiar $\mathrm{F}$ star $\gamma$ Equ (see Fig. 12 middle and right panels). In the case of AD Leo, the Stokes $V$ LSD signature we detect in a $4 \times 61$-s polarization sequence (with $\mathrm{SNR}=360$ per pixel in $\mathrm{H})$ yields a longitudinal magnetic field of $-135 \pm 10 \mathrm{G}$, significantly smaller than the one measured (and mapped) a decade ago with ESPaDOnS observations (e.g., Morin et al. 2008) and consistent with recent findings that the magnetic field of this star has been regularly decreasing with time over the last decade (Lavail et al. 2018). We note in particular that the Stokes $I$ and $V$ profiles, spreading over a velocity 

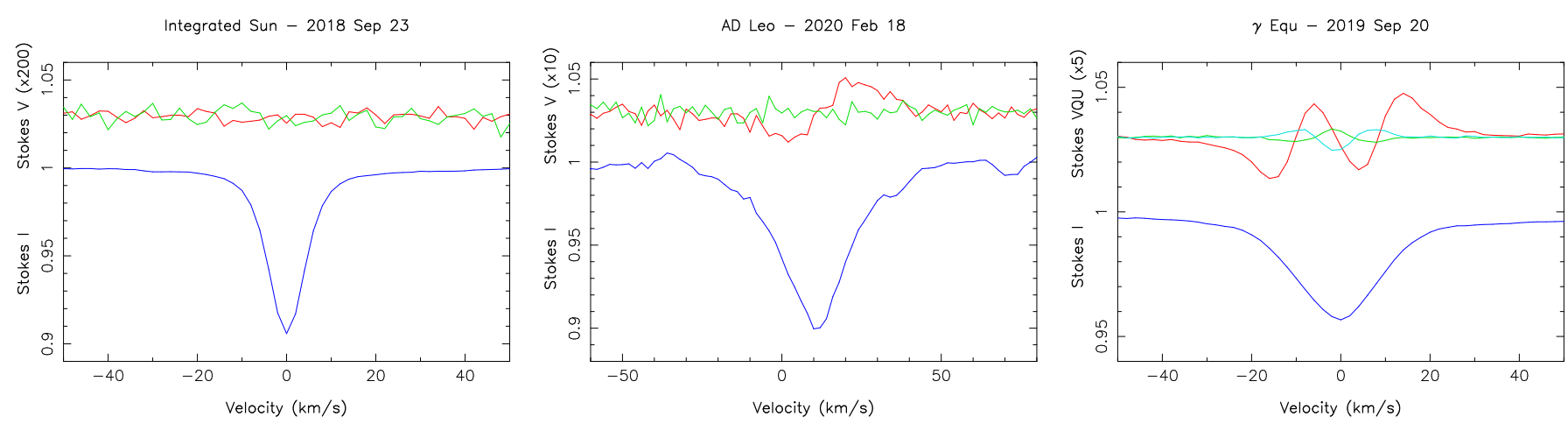

Figure 12. Polarimetric sensitivity of SPIRou at a precision of $10 \mathrm{ppm}$, as estimated from the observation of the integrated Sun (left panel). Observing the magnetic stars AD Leo and $\gamma$ Equ with SPIRou yields magnetic detections consistent with expectations in both circular and linear polarization (middle and right panels), and demonstrates that crosstalk between both polarization states is nominal. Stokes $I$ and $V$ LSD profiles are shown as blue and red lines respectively, whereas Stokes $Q$ and $U$ LSD profiles (of $\gamma$ Equ) are shown in green and cyan. All polarized LSD profiles are shifted upwards (by 1.03) and expanded (by a different factor for each star) for display purposes. In the left and middle panel, the null polarization LSD profile is also shown (in green, with the same shift and expansion factor as those of the Stokes $V$ LSD profiles) to emphasize that no spurious polarization signal is present.

range of at least $50 \mathrm{~km} \mathrm{~s}^{-1}$, are significantly broader and shallower than their optical counterparts (spanning no more than $40 \mathrm{~km} \mathrm{~s}^{-1}$, Morin et al. 2008; Lavail et al. 2018) as a result of the larger Zeeman broadening at nIR wavelengths.

SPIRou also very clearly detects circular and linear polarization Zeeman signatures in the lines of $\gamma \mathrm{Equ}$, which can be used to accurately estimate SPIRou's crosstalk level between polarization states (through measurements carried out at various Bonnette angles). We find that crosstalk from Stokes $V$ to Stokes $Q$ and Stokes $U$ polarizations was respectively equal to $1.2 \%$ and $1.1 \%$ at the time of our observations, in agreement with the specification $(<2 \%)$.

\section{OVERVIEW OF FIRST RESULTS}

In this penultimate section, we present a quick overview of the very first results obtained with SPIRou from data secured during commissionning and the beginning of the SLS. We stress that the goal of this paper is not to carry out detailed analyses on any single data set discussed below (to be presented in future papers from members of the SLS team, e.g., Moutou et al. 2020; Martioli et al. 2020), but rather to highlight the potential of SPIRou with a number of examples taken from most science topics that SPIRou will tackle (in particular those of the SLS, see Sec. 2).

\subsection{Detecting \& characterizing exoplanets of $M$ dwarfs}

We start this panorama with a small sample of 13 observations of the M3 dwarf Gl 436, known to host a transiting warm Neptune on an inclined, eccentric orbit (Lanotte et al. 2014). This data set was obtained with SPIRou during a 16$\mathrm{n}$ run in the second half of 2019 April (see Fig. 13). The measured RVs from the $134 \times 245$-s polarization sequences (with a median SNR of 300 per pixel in $\mathrm{H}$ ) collected on Gl 436 are found to be in good agreement with the published ephemeris, with an RMS dispersion about the predicted RV curve of $2.6 \mathrm{~m} \mathrm{~s}^{-1}$ if assuming equal weights for all points, and $2.3 \mathrm{~m} \mathrm{~s}^{-1}$ with a weight equal to the inverse variance of
RVs within each polarization sequence; the internal dispersion of RVs between individual exposures ranges from 1.9 to $5.0 \mathrm{~m} \mathrm{~s}^{-1}$ from night to night, with a median of $2.9 \mathrm{~m} \mathrm{~s}^{-1}$, similar to the internal RV precision obtained within polarization sequences of the inactive dwarf Gl 699 (see Sec. 4). The dispersion of RV residuals we report for this star is consistent with the RV precision of $\simeq 2 \mathrm{~m} \mathrm{~s}^{-1}$ RMS found on other quiet $\mathrm{M}$ dwarfs (see Fig. 11 middle and right panels), and larger than both the expected photon noise level (of $0.5 \mathrm{~m} \mathrm{~s}^{-1}$ ) and of the RV precision (of $1 \mathrm{~m} \mathrm{~s}^{-1}$ ) SPIRou aims at, suggesting that there is still room for improvements. No magnetic field is detected at the surface of this (relatively inactive) dwarf, down to a precision of about $3 \mathrm{G}$. A lot more results of this type are expected in the framework of the SLS-PS and SLS-TF (see Sec. 2), thanks to which new planets of nearby $\mathrm{M}$ dwarfs will be discovered or confirmed, and characterized (e.g., AU Mic b, Klein et al., 2020a, submitted).

\subsection{Characterizing transiting exoplanets \& their atmospheres}

As part of the SLS, one full transit of the hJ of the K2 star HD 189733 was monitored with SPIRou on 2019 June 15 , with $50 \times 250$-s exposures in a fixed rhomb configuration and a median SNR of 230 per pixel in H. The RossiterMcLaughlin effect is easily detected and consistent with the published literature, whereas the RV precision throughout the transit is found to be $5.3 \mathrm{~m} \mathrm{~s}^{-1}$ RMS $\left(4.3 \mathrm{~m} \mathrm{~s}^{-1}\right.$ RMS on the out-of-transit points, see Fig. 13 bottom panel), larger than the photon noise level (of $1.8 \mathrm{~m} \mathrm{~s}^{-1}$ on average) and in line with the activity jitter of this (moderately active) star. A detailed analysis of these transit data, of additional data from another transit (2018 September 22), as well as spectropolarimetric data (with detected magnetic fields of a few G) collected over several rotation cycles of HD 189733 and orbital cycles of its hJ, are presented in Moutou et al. (2020), whereas a similar study on the young M dwarf AU Mic was recently completed (Martioli et al. 2020).

SPIRou is also a powerful instrument for investigating exoplanet atmospheres through transmission or reflec- 
GI 436 - 2019 April 15-May 01
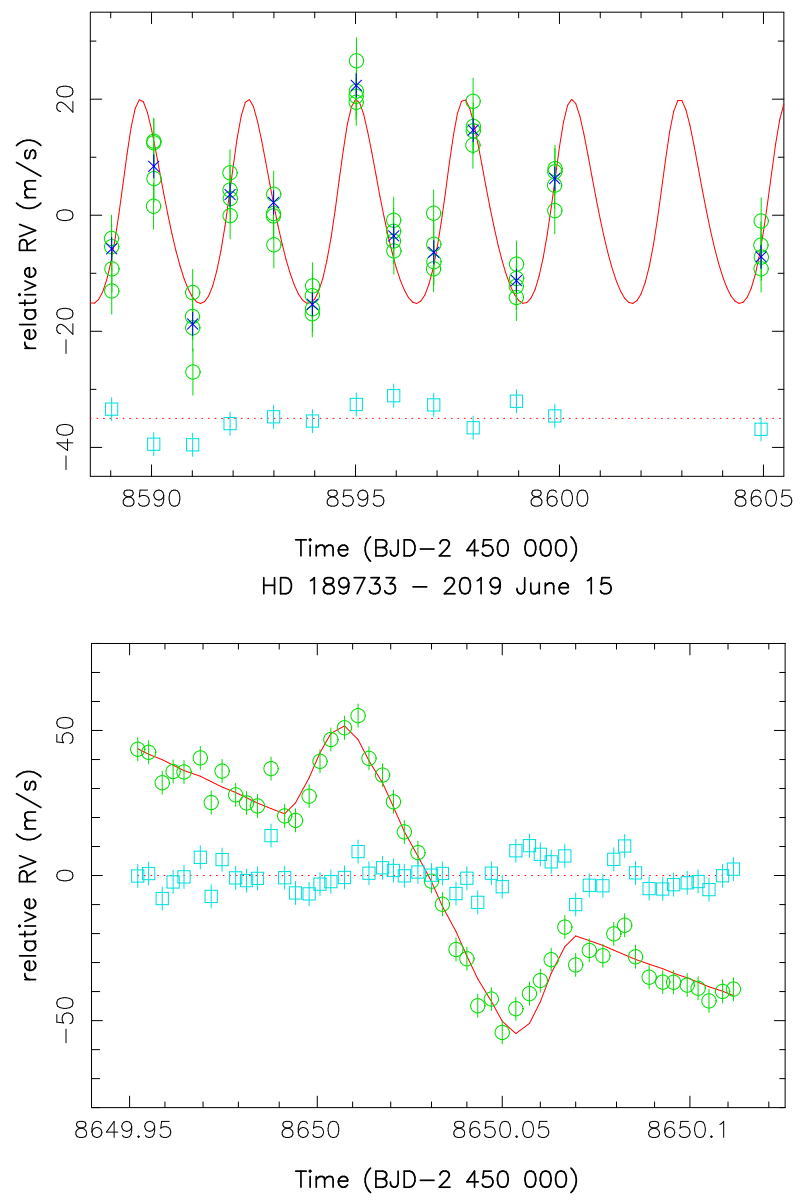

Figure 13. RV measurements for the planet-hosting M3 dwarf Gl 436 (top panel) and for the K2 dwarf HD 189733 (bottom). The RMS dispersion with respect to the published ephemeris of Gl 436 (Lanotte et al. 2014) (red line) is equal to $2.6 \mathrm{~m} \mathrm{~s}^{-1}$ for the RVs derived from polarimetric sequences (blue crosses, with residuals, shown as cyan squares, shifted by $-35 \mathrm{~m} \mathrm{~s}^{-1}$ for clarity). The median dispersion of RVs measured from individual exposures within polarization sequences (green circles) is equal to $2.9 \mathrm{~m} \mathrm{~s}^{-1}$, and the RMS dispersion of the main RV measurements reaches $2.3 \mathrm{~m} \mathrm{~s}^{-1}$ when weighting each RV point by the inverse variance within each polarization sequence. In the case of HD 189733, observed with a fixed rhomb configuration, the RMS dispersion of the residuals (cyan squares) with respect to the Rossiter-McLaughlin signature model (red line) of Moutou et al. (2020) is equal to $5.3 \mathrm{~m} \mathrm{~s}^{-1}$, and $4.3 \mathrm{~m} \mathrm{~s}^{-1}$ on the out-of-transit points. As in Fig. 11, error bars are set to $4 \mathrm{~m} \mathrm{~s}^{-1}$ for the points corresponding to individual exposures (green circles in both panels and cyan squares in bottom panel), and to $2 \mathrm{~m} \mathrm{~s}^{-1}$ for those associated with polarization exposures (blue crosses and cyan squares in top panel).

tion spectroscopy (Brogi et al. 2012, 2016), thanks to the extra-wide spectral domain recordable in a single exposure and the associated multiplex gain (Brogi et al. 2018; Brogi \& Line 2019), making it at least as (if not more) efficient for such applications than CRIRES on the VLT, even in its updated version CRIRES+. For instance, the $36 \times 250$-s spectra of HD 189733 collected with SPIRou on 2018 September 22 reveal increased He I absorption during the transit of its hJ (see Fig. 14) with a signature similar to those detected with
HD 189733 - 2018 September 22 - 1083-nm He I line

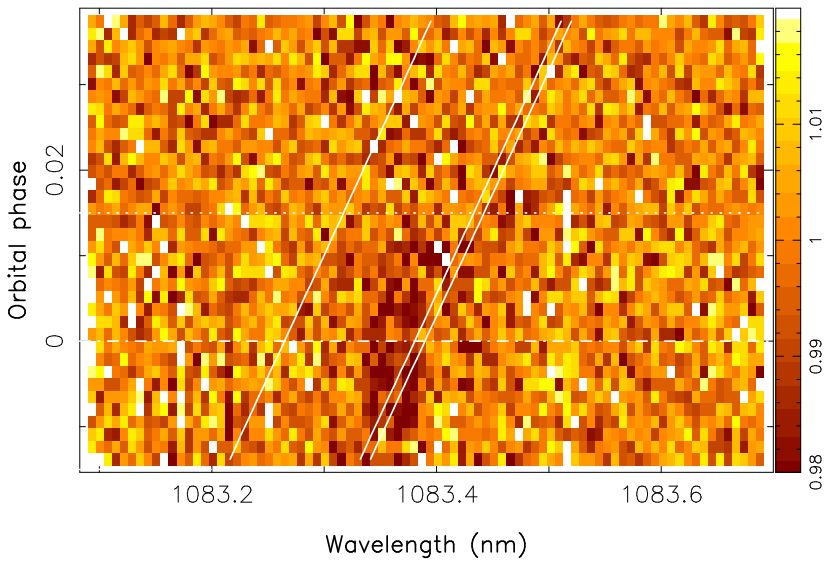

Figure 14. SPIRou spectra of HD 189733 around the $1083 \mathrm{~nm}$ He I line during the transit of the hJ, after normalizing all spectra by the median out-of-transit spectrum. The slanted full lines trace the velocity curve of the planet for each of the He I triplet component, whereas the horizontal dash-dot and dotted lines respectively depict the orbital phases of mid transit and egress. A patch of increased He I absorption is detected at mid transit in association with the 2 main (red) components of the He I $1083 \mathrm{~nm}$ triplet (about an order of magnitude deeper than the third blue component).

Carmenes (Salz et al. 2018), whereas the other transit monitored with SPIRou (on 2019 June 15) shows a much weaker absorption signal (if any). We also note that the detected signature does not closely follow the planet trajectory (depicted by a slanted line on Fig. 14), as for the second transit reported in Salz et al. (2018); this suggests that the detected He I signature may at least partly be attributable to stellar activity, e.g., when chromospheric regions are masked by the transiting planet. This detection of He I absorption and its temporal variability illustrates the potential of such observations to investigate extended atmospheres of exoplanets and their possible evolution on timescales of a few months. Looking for molecular species such as $\mathrm{H}_{2} \mathrm{O}$ and $\mathrm{CO}_{2}$ is also promising, for diagnosing elemental abundances and ratios such as $[\mathrm{C} / \mathrm{O}]$ bringing key constraints for theoretical models. Studies from SPIRou data on these topics are currently being carried out for a number of transiting exoplanets (Darveau-Bernier et al. 2020; Boucher et al. 2020; Klein et al. 2020b, in prep).

\subsection{Magnetic fields \& accretion / ejection processes of TTSs}

Looking at TTSs in the context of the SLS-MP, SPIRou routinely detects polarized Zeeman signatures in their spectral lines, probing the presence of strong magnetic fields at their surfaces. For instance, large Zeeman signatures are detected in conjunction with atomic lines in the spectrum of the very young wTTS V410 Tau (see Fig. 15 top panel for the Stokes $I$ and $V$ profiles of a typical $4 \times 300$-s polarization sequence with a SNR of 200 per pixel in $\mathrm{H}$, and bottom panel for the full data set collected on this star in 2019 November and December), with multiple lobes of opposite signs revealing a complex magnetic field at the surface of the star. The cor- 
V410 Tau - 2019 Nov 11
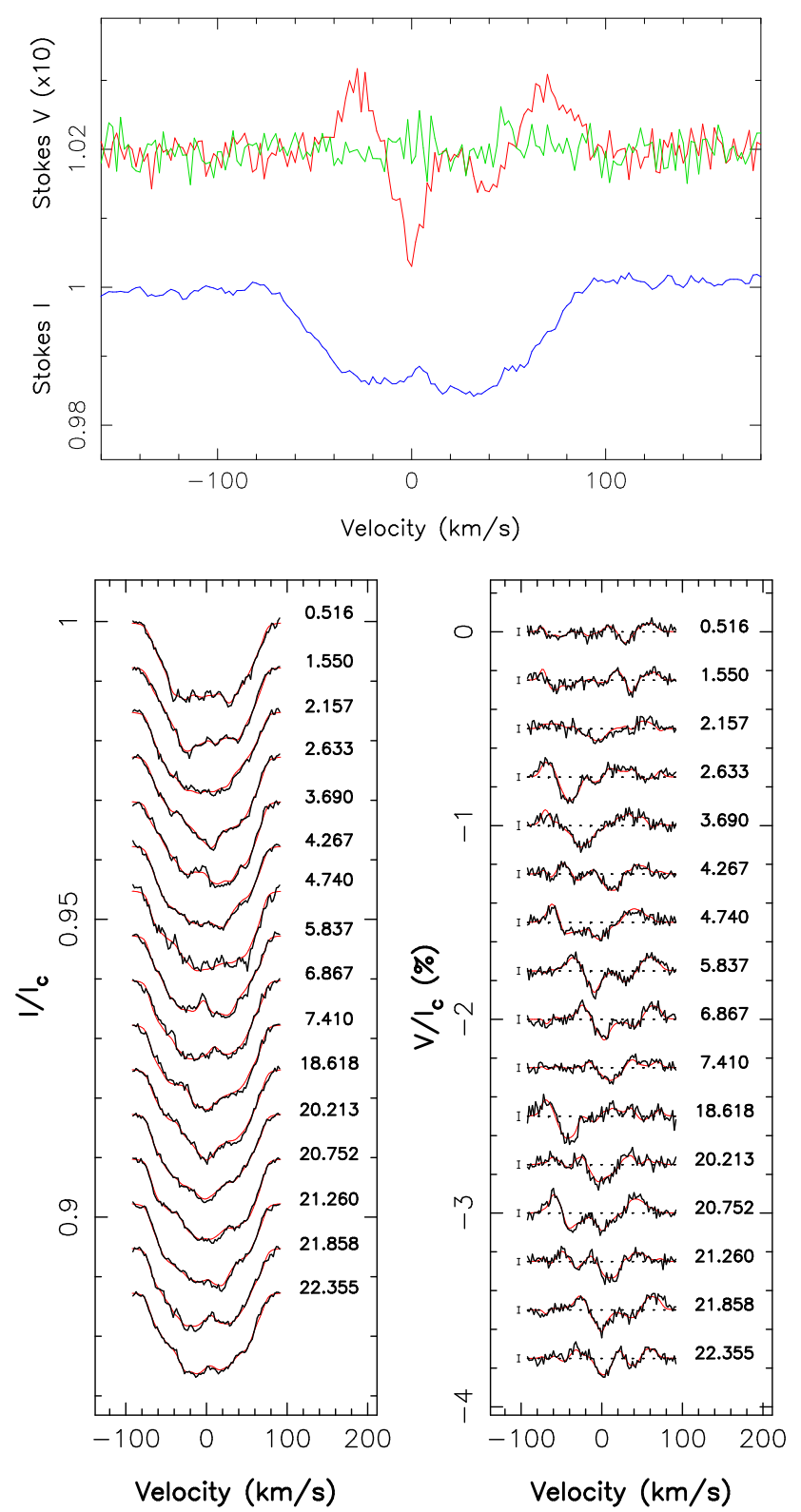

Figure 15. LSD Stokes $I$ and $V$ profiles of the wTTS V410 Tau on 2019 Nov 11 (top panel, blue and red curves respectively, with the null polarization profile also shown in green) showing obvious Zeeman detections in conjunction with photospheric lines probing the presence of strong magnetic fields at the surface of the young PMS star, and full set of LSD Stokes $I$ and $V$ profiles collected with SPIRou in 2019 November \& December (bottom panels, thick black line) along with the ZDI fit (thin red line) to these observations (with rotational phases indicated next to each LSD profile, and a $\pm 1 \sigma$ error bar shown for each Zeeman signature).

responding longitudinal fields are found to vary from 40 to $-240 \mathrm{G}$ with typical error bars of $30 \mathrm{G}$, i.e., similar to those reported from ESPaDOnS spectra (Yu et al. 2019) but with a smaller error bar in half the exposure time. It demonstrates the gain brought by observing PMS stars at nIR wavelengths and thereby the improved sensitivity of SPIRou for investigating magnetic fields of young stars.
The preliminary brightness and magnetic maps derived from this first SPIRou data set on V410 Tau (see lower panel of Fig. 15 and Fig. 16) are similar to those obtained from optical data (Yu et al. 2019), though with less contrasted surface brightness inhomogeneities (as expected for cool features of low-mass stars observed in the nIR); in particular, the cool spot complex repeatedly reconstructed close to the pole from optical data, is much less conspicuous (if even present) in this new map from SPIRou data. The inferred magnetic topology is also quite consistent with the findings of Yu et al. (2019), with a (mainly non-axisymmetric) poloidal component enclosing $65 \%$ of the total magnetic energy and a dipole component with a polar field strength of $400 \mathrm{G}$ (tilted at $20^{\circ}$ to the rotation axis). A detailed analysis of these data is being carried out, to be published soon (Finociety et al. 2020, in prep).

The nIR domain also features a number of interesting new proxies for studying accretion and ejection processes taking place in the circumstellar environments of cTTSs, which are key for addressing the main goals of the SLS regarding star / planet formation (see Sec. 2). More specifically, these proxies are being used to monitor the inner regions of their accretion discs and the magnetospheric gaps the intense magnetic fields of these young stars are able to carve at disc center, where complex time-variable patterns of inflows and outflows are occurring. We show in Fig. 17 (bottom panel) the example of the 1083-nm He I line of the well-known cTTS CI Tau, whose accretion disc is thought to be the location of active planet formation (Clarke et al. 2018). This line is known to be a powerful tracer for investigating both outflows and inflows through its blue-shifted absorption and red-shifted emission components respectively (Edwards 2009). In the case of CI Tau, the variability of both components is obvious, e.g., with the blue-shifted absorption moving in position and strength with time and even disappearing entirely at some epochs. By making it possible to connect the main characteristics of this variability with a detailed modeling of the magnetospheric topologies of cTTSs (as in, e.g., Donati et al. 2020), SLS-MP studies will bring new insight for our understanding of magnetospheric accretion / ejection processes of cTTSs, especially for younger, lower-mass and more strongly accreting PMS stars than previously possible.

\subsection{Stellar atmospheres, activity \& Earth's atmosphere}

As pointed out in Sec. 2, there are many more science goals that SPIRou can investigate beyond those on which the SLS is focusing. An obvious one, on which the SLS is also contributing to as a legacy output, is the study of the atmospheres of M dwarfs from their high-resolution nIR spectra, which already motivated some attention and triggered new developments with the Phoenix model atmospheres (Rajpurohit et al. 2013; Allard et al. 2013; Rajpurohit et al. 2018a,b). Thanks to the extended domain and the high spectral resolution, SPIRou spectra are particularly adapted for such studies; a straightforward comparison of the closest synthetic Phoenix spectrum (extracted from the database of Husser et al. 2013) matching the atmospheric parameters of Gl $699\left(T_{\text {eff }}=3200 \mathrm{~K}, \log g=5.0,[\mathrm{Fe} / \mathrm{H}]=-0.5\right.$, e.g., Mann et al. 2015) and broadened to the spectral resolution 

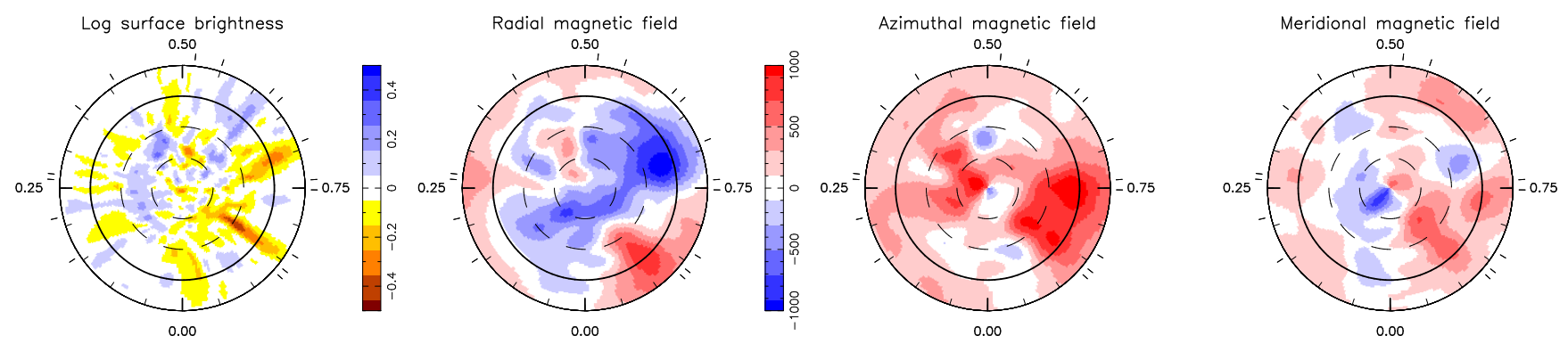

Figure 16. Preliminary ZDI map of the logarithmic relative surface brightness (left panel), and the radial, meridional and azimuthal magnetic field (second to fourth panels) of V410 Tau, reconstructed from the data set shown in the bottom panels of Fig. 15. The star is shown in a flattened polar projection, with the equator depicted as a bold circle and the $30^{\circ}$ and $60^{\circ}$ latitude parallels as dashed lines. Ticks around the star mark the rotational phases of our observations.

Cl Tau - 2019 October 05-16 - 1083-nm He I line

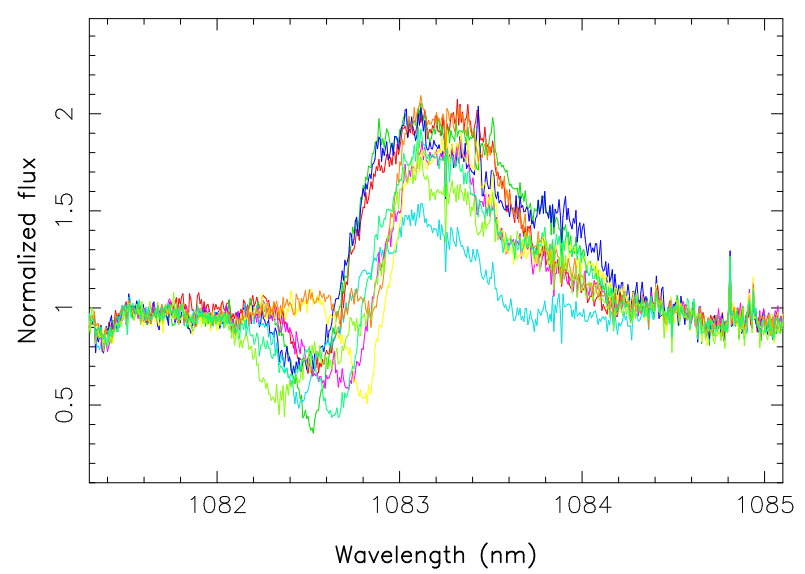

Figure 17. Temporal evolution of the 1083-nm He I line of the cTTS CI Tau over the 2019 October 02-15 SPIRou run showing variability of both blue absorption and red emission components probing accretion and ejection processes taking place in the close circumstellar environment.

of SPIRou shows that, although the main lines of the observed spectrum agree with model predictions, obvious discrepancies remain and abundant spectral content is absent from the model (see Fig. 18). Ongoing studies from SPIRou data are being carried out in this field, including within the SLS.

Studies of stellar activity, complementing those on precise RVs (to improve the efficiency at filtering RV curves) and magnetometry of late-type dwarfs (to study how activity relates to and fuels from the magnetic field that produces it), will also benefit from SPIRou and the new activity diagnostics that are available in the nIR domain (e.g., Schöfer et al. 2019), in particular He I (see Figs. 14 and 17), $\mathrm{Pa} \beta$, $\operatorname{Pa} \gamma$ and $\operatorname{Br} \gamma$. Measuring surface magnetic fluxes from smallscale fields (generating little to no polarization signatures as a result of their tangled structures) thanks to the stronger Zeeman splitting at nIR wavelengths will be quite valuable in this respect, as this proxy was shown to be the one that correlates best with RV variations in the case of the Sun (Haywood et al. 2016). Papers based on (or using) SPIRou data are being worked on, with studies on, e.g., the activity and magnetic field of $\epsilon$ Eri as seen by Narval, SPIRou and K2 (Petit et al. 2020, in prep).
Last but not least, SPIRou is an ideal tool for studying column densities of specific molecules in the Earth's atmosphere, in particular $\mathrm{H}_{2} \mathrm{O}, \mathrm{CO}_{2}, \mathrm{CH}_{4}$ and $\mathrm{O}_{2}$ that exhibit a rich absorption spectrum in the SPIRou domain, and their evolution with time on both short- and long-term. An example of such modeling is given in Fig. 18 (bottom panel), showing the atmospheric content of $\mathrm{H}_{2} \mathrm{O}, \mathrm{CO}_{2}$ and $\mathrm{CH}_{4}$ as a function of time over the $6 \mathrm{hr}$ monitoring of Gl 514 with SPIRou on 2019 May 14 (see Fig. 11 middle panel), and derived by adjusting TAPAS atmospheric transmission models (Bertaux et al. 2014) to SPIRou data. This example illustrates that, whereas $\mathrm{CH}_{4}$ and $\mathrm{CO}_{2}$ are strongly correlated with airmass (being evenly distributed in the atmosphere), $\mathrm{H}_{2} \mathrm{O}$ is much more variable and subject to (more rapidly varying) weather conditions. This kind of analysis can be used not only to quantitatively optimize in almost real time ongoing SPIRou observations given current weather conditions, but also to significantly contribute, in the context of global warming, to the long-term monitoring of the Earth's evolving atmosphere in the best temperate astronomical site worldwide.

\section{SUMMARY AND PERSPECTIVES}

In this paper, we first present the wide variety of science goals for which SPIRou was constructed; we focus in particular on the two main ones, namely the quest for planetary systems around nearby $M$ dwarfs and the study of magnetized star / planet formation, on which the SLS concentrates through a dedicated 300-night Large Programme at the CFHT. We then detail the main modules the instrument consists of, starting with the Cassegrain unit and its achromatic polarimeter mounted on the telescope, the fiber link conveying the stellar light to the CFHT Coudé room, where the cryogenic bench-mounted cross-dispersed highresolution spectrograph is mounted along with the calibration unit providing the instrument with spectra of reference calibration lamps. We also provide basic information on the automatic reduction pipeline APERO, including its telluric correction process.

Next, we outline the current instrument performances as estimated from both lab tests and on-sky observations. SPIRou yields stellar spectra covering $0.95-2.50 \mu \mathrm{m}$ in a single exposure, with a sampling rate of $2.28 \mathrm{~km} \mathrm{~s}^{-1}$ per pixel and a spectral resolving power of $70 \mathrm{k}$ (in terms of the broad- 
Observed vs synthetic spectrum of G| 699

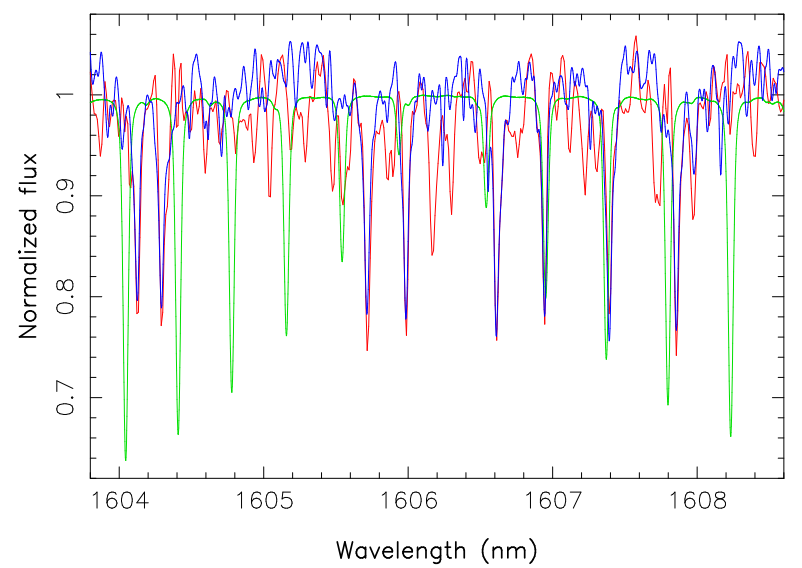

Atmospheric content of $\mathrm{H}_{2} \mathrm{O}, \mathrm{CO}_{2} \& \mathrm{CH}_{4}$

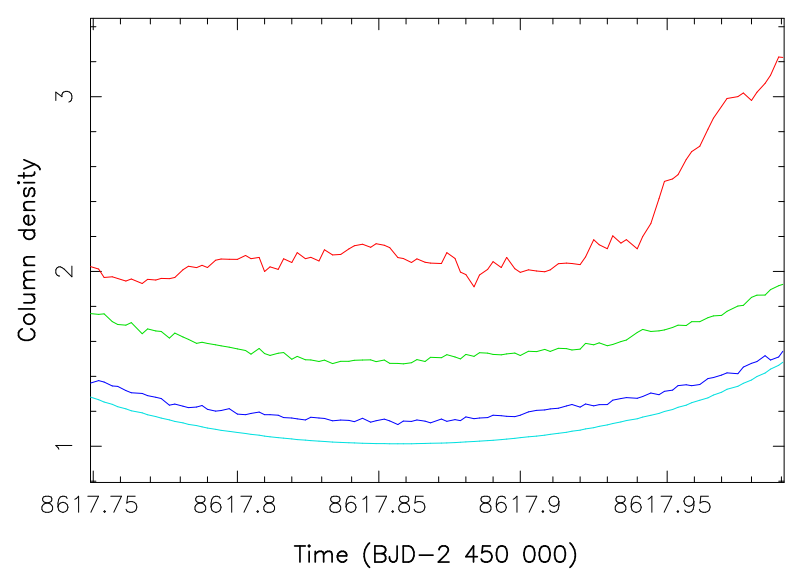

Figure 18. Top panel: Small portion of the (telluric corrected) SPIRou spectrum of the M4 dwarf Gl 699 (red) compared with the (broadened) synthetic high-resolution spectrum in the Phoenix database of Husser et al. (2013) that matches best the atmospheric parameters of Gl 699 (blue), along with a TAPAS spectrum for average atmospheric conditions at the CFHT (green), found to provide a very accurate description of the true telluric features over most of the SPIRou domain including the one shown here. Whereas the main lines in this spectral window are in reasonable agreement, significant spectral content in the SPIRou observation is absent from the model. Bottom panel: $\mathrm{H}_{2} \mathrm{O}$ (red), $\mathrm{CO}_{2}$ (green) and $\mathrm{CH}_{4}$ (blue) atmospheric content as a function of time throughout the 6-hr SPIRou monitoring of Gl 514 (see Fig. 11 middle panel), as derived with a TAPAS model of atmospheric transmission (Bertaux et al. 2014) fitted to the SPIRou data. Airmass is also shown (cyan).

ening impact on stellar spectra). Throughput rises from $3 \%$ in the $\mathrm{Y}$ band, to $7 \%, 10 \%$ and $12 \%$ in the $\mathrm{J}, \mathrm{H}$ and $\mathrm{K}$ band respectively, making it capable of collecting spectra of $M$ dwarfs with peak SNRs of 110 per pixel at $\mathrm{H} \simeq 8$ in a 5 -min exposure in good weather conditions. Thermal background at $2.35 \mu \mathrm{m}$ is currently equivalent to the flux of a $\mathrm{H} \simeq 8.6$ mid-M dwarf, with plans to reduce it in the near future. RV precision is currently found to be $\simeq 2 \mathrm{~m} \mathrm{~s}^{-1} \mathrm{RMS}$ on a timescale of at least a few weeks, reaching at times $1 \mathrm{~m} \mathrm{~s}^{-1}$ RMS on bright stars in good weather, and is expected to further improve with forthcoming hardware and software (APERO) upgrades. Polarimetric performances are similar to those of ESPaDOnS, with a sensitivity of at least $10 \mathrm{ppm}$ and a crosstalk level from circular to linear polarization of order $1 \%$.

We finally present a quick overview of a few basic results obtained so far with SPIRou, which are (or will soon be) described in detail in dedicated papers. We show in particular that SPIRou is able to detect the RV impact of a known exoplanet (Gl 436b), the Rossiter McLaughlin effect of a transiting hJ (HD 189733b), the He I absorption from the atmosphere of a transiting hJ (again HD 189733b), and the Zeeman signatures from surface magnetic fields of a $M$ dwarf (AD Leo) and a TTS (V410 Tau). We also illustrate how SPIRou contributes to studies of stellar atmospheres and of the Earth's atmosphere.

At this time, SPIRou has been carrying out science programmes, including the SLS, at the CFHT since the beginning of 2019. SPIRou is already the most popular CFHT instrument, capitalizing the largest number of PI proposals since it has been offered to the whole community. SPIRou programmes were allocated a total of 209 nights on the CFHT in the first 3 semesters of science operation (2019a to 2020a). Data collection has however progressed more slowly than expected due to a number of unfortunate issues (from poor weather to technical failures at the CFHT, TMT protests in Hawaii, and lately the COVID-19 pandemic) that altogether caused the loss of almost half the allocated SPIRou time so far. SPIRou being mounted in bright time periods only also comes as a limitation, in particular for the observation of transiting planets with orbital periods longer than a week. The situation will hopefully improve in the coming semesters, with more SPIRou time allocated to ensure faster progression and better completion of ongoing programmes.

Over the longer term, SPIRou is expected to be a key contributor in coordinated programmes with most major facilities to come, in particular the JWST from 2021, the ELTs from 2025, then PLATO from 2026 and ARIEL from 2028. More specifically, SPIRou velocimetric and spectropolarimetric observations will be instrumental for identifying the most promising nearby Earth-like HZ planets to be scrutinized by the JWST and the ELTs for detailed atmospheric characterization, and for providing consistent modelings of the star / disc / planet interactions taking place at the heart of forming star / planet systems. Later on, SPIRou will be needed for confirming and characterizing planetary systems around M stars to be unveiled by PLATO, and for monitoring and modeling the activity of stars to be investigated with ARIEL. Altogether, it requires SPIRou to progressively ramp up in duty cycle so that it can achieve very dense temporal monitoring for the large stellar samples to be observed in coordination with the major facilities of the decade. Coupling SPIRou with ESPaDOnS so that both instruments can observe the same targets at the same time and provide highresolution spectra of the observed stars all the way from 0.39 to $2.50 \mu \mathrm{m}$ in a single exposure for any given polarization state, would further boost the overall science return and make the CFHT the only observatory worldwide offering such capabilities. 


\section{ACKNOWLEDGEMENTS}

This paper is dedicated to the memory of Les Saddlemyer from NRC-H, who managed the development of the SPIRou dewar, and to Laurent Parès from OMP/IRAP in charge of the optical design and tests of the Cassegrain unit, who sadly passed away in 2017 January and 2020 February, respectively.

We thank all partners for funding SPIRou, whose construction cost (including reviews and travels) reached $\simeq 5 \mathrm{M} €$, namely the IDEX initiative at UFTMP, UPS, the DIM-ACAV programme in Région Ile de France, the MIDEX initiative at AMU, the Labex@OSUG2020 programme, UGA, INSU/CNRS, CFI, CFHT, LNA, DIAS, ASIAA and IA. We are grateful for inkind manpower allocated to SPIRou by OMP/IRAP, OHP/LAM, IPAG, CFHT, NRC-H, UdeM, UL, OG, LNA and ASIAA, amounting to about 75 FTEs including installation and ongoing upgrades. We also thank an anonymous referee for valuable comments that improved the manuscript.

JFD and TPR acknowledge funding from the European Research Council under the H2020 research \& innovation programme (grants \#740651 NewWorlds and \#743029 EASY). We also acknowledge funding from the French "Agence Nationale pour la Recherche" (grants ANR-18-CE31-0019 and ANR-15IDEX-02). This work was supported by the Fundação para a Ciẽncia e a Tecnologia and FEDER through COMPETE2020 (UID/FIS/04434/2019, UIDB/04434/2020, UIDP/04434/2020, PTDC/FIS-AST/32113/2017, POCI-01-0145-FEDER-032113, PTDC/FIS-AST/28953/2017, POCI-01-0145-FEDER-028953). SYW acknowledges funding from ASIAA.

Our study is based on data obtained at the CFHT, operated by the CNRC (Canada), INSU/CNRS (France) and the University of Hawaii. The authors wish to recognise and acknowledge the very significant cultural role and reverence that the summit of Maunakea has always had within the indigenous Hawaiian community. We are most fortunate to have the opportunity to conduct observations from this mountain. This work also benefited from the TAPAS service through the ETHER center at URL http://ether.ipsl.jussieu.fr/tapas/, the SIMBAD / CDS database at URL http://simbad.u-strasbg.fr/simbad and the ADS system at URL https://ui.adsabs.harvard.edu.

\section{DATA AVAILABILITY}

Most data underlying this article are part of the SLS, and will be publicly available from the Canadian Astronomy Data Center 1 yr following the completion of the SLS. Data on RV and telluric standards are already public.

\section{REFERENCES}

Alencar S. H. P., et al., 2018, A\&A, 620, A195

Allard F., Homeier D., Freytag B., Schaffenberger W., Rajpurohit A. S., 2013, Memorie della Societa Astronomica Italiana Supplementi, 24, 128

André P., Basu S., Inutsuka S., 2009, The formation and evolution of prestellar cores. Cambridge University Press, p. 254

Artigau É., Bouchard S., Doyon R., Lafrenière D., 2009, ApJ, 701,1534

Artigau É., et al., 2012, SPIRou @ CFHT: data reduction software and simulation tools. p. 84513I, doi:10.1117/12.926334

Artigau É., et al., 2014, in Ground-based and Airborne Instrumentation for Astronomy V. p. 914715 (arXiv:1406.6992), doi:10.1117/12.2055663

Artigau É., Saint-Antoine J., Lévesque P.-L., Vallée P., Doyon R., Hernandez O., Moutou C., 2018, in Proc. SPIE. p. 107091P, doi:10.1117/12.2314475
Baraffe I., Chabrier G., 2010, A\&A, 521, A44

Barclay T., Pepper J., Quintana E. V., 2018, ApJS, 239, 2

Barrick G. A., et al., 2012, in Software and Cyberinfrastructure for Astronomy II. p. 84513J, doi:10.1117/12.926392

Barrick G., et al., 2018, in Proc. SPIE. p. 1070268, doi: $10.1117 / 12.2312317$

Baruteau C., et al., 2014, Protostars and Planets VI, pp 667-689

Bertaux J. L., Lallement R., Ferron S., Boonne C., Bodichon R., 2014, A\&A, 564, A46

Boisse I., Perruchot S., Bouchy F., Dolon F., Moreau F., Sottile R., Wildi F., 2016, in Society of Photo-Optical Instrumentation Engineers (SPIE) Conference Series. p. 990868 (arXiv: 1612.03679), doi:10.1117/12.2231678

Bonfils X., et al., 2013, A\&A, 549, A109

Bouchy F., Doyon R., Pepe F., Wildi F., Blind N., Artigau E., Malo L., 2019, in EPSC-DPS Joint Meeting 2019. pp EPSCDPS2019-1860

Bouvier J., 2017, Mem. Soc. Astron. Italiana, 88, 587

Bouvier J., Alencar S. H. P., Harries T. J., Johns-Krull C. M., Romanova M. M., 2007, in Reipurth B., Jewitt D., Keil K., eds, Protostars and Planets V. pp 479-494

Brogi M., Line M. R., 2019, AJ, 157, 114

Brogi M., Snellen I. A. G., de Kok R. J., Albrecht S., Birkby J., de Mooij E. J. W., 2012, Nature, 486, 502

Brogi M., de Kok R. J., Albrecht S., Snellen I. A. G., Birkby J. L., Schwarz H., 2016, ApJ, 817, 106

Brogi M., Giacobbe P., Guilluy G., de Kok R. J., Sozzetti A., Mancini L., Bonomo A. S., 2018, A\&A, 615, A16

Cersullo F., Wildi F., Chazelas B., Pepe F., 2017, A\&A, 601, A102

Challita Z., et al., 2018, in Proc. SPIE. p. 1070262, doi:10.1117/12.2311999

Cirasuolo M., 2019, in The Very Large Telescope in 2030. p. 16, doi:10.5281/zenodo.3356242

Clarke C. J., et al., 2018, ApJ, 866, L6

Cloutier R., et al., 2018, AJ, 155, 93

Cloutier R., et al., 2019, A\&A, 629, A111

Cody A. M., et al., 2014, AJ, 147, 82

Cosby P. C., Sharpee B. D., Slanger T. G., Huestis D. L., Hanuschik R. W., 2006, Journal of Geophysical Research (Space Physics), 111, A12307

Crossfield I. J. M., et al., 2014, Nature, 505, 654

David T. J., et al., 2016, Nature, 534, 658

David T. J., Petigura E. A., Luger R., Foreman-Mackey D., Livingston J. H., Mamajek E. E., Hillenbrand L. A., 2019, ApJ, 885, L12

Davies C. L., Gregory S. G., Greaves J. S., 2014, MNRAS, 444, 1157

Donati J.-F., 2003, in Trujillo-Bueno J., Sanchez Almeida J., eds, Astronomical Society of the Pacific Conference Series Vol. 307, Astronomical Society of the Pacific Conference Series. p. 41

Donati J., Landstreet J. D., 2009, ARA\&A, 47, 333

Donati J.-F., Semel M., Carter B. D., Rees D. E., Collier Cameron A., 1997, MNRAS, 291, 658

Donati J.-F., Paletou F., Bouvier J., Ferreira J., 2005, Nature, 438,466

Donati J.-F., et al., 2012, MNRAS, 425, 2948

Donati J.-F., et al., 2017, MNRAS, 465, 3343

Donati J.-F., et al., 2018, SPIRou: A NIR Spectropolarimeter/High-Precision Velocimeter for the CFHT. p. 107, doi:10.1007/978-3-319-55333-7_107

Donati J. F., et al., 2020, MNRAS, 491, 5660

Dressing C. D., Charbonneau D., 2015, ApJ, 807, 45

Edwards S., 2009, in Stempels E., ed., American Institute of Physics Conference Series Vol. 1094, 15th Cambridge Workshop on Cool Stars, Stellar Systems, and the Sun. pp 29-38 (arXiv:0809.3603), doi:10.1063/1.3099106

Emeriau-Viard C., Brun A. S., 2017, ApJ, 846, 8 
Feiden G. A., 2016, A\&A, 593, A99

Flores C., Connelley M. S., Reipurth B., Boogert A., 2019, ApJ, 882,75

Fouqué P., et al., 2018, MNRAS, 475, 1960

Gaidos E., Mann A. W., Kraus A. L., Ireland M., 2016, MNRAS, 457,2877

Gillon M., et al., 2017, Nature, 542, 456

Gregory S. G., Donati J.-F., Morin J., Hussain G. A. J., Mayne N. J., Hillenbrand L. A., Jardine M., 2012, ApJ, 755, 97

Güdel M., et al., 2014, Protostars and Planets VI, pp 883-906

Gullikson K., Dodson-Robinson S., Kraus A., 2014, AJ, 148, 53

Gully-Santiago M. A., et al., 2017, ApJ, 836, 200

Haywood R. D., et al., 2016, MNRAS, 457, 3637

Hébrard É. M., Donati J.-F., Delfosse X., Morin J., Moutou C., Boisse I., 2016, MNRAS, 461, 1465

Hennebelle P., Commerçon B., Lee Y.-N., Charnoz S., 2020, A\&A, 635, A67

Hill C. A., Folsom C. P., Donati J. F., Herczeg G. J., Hussain G. A. J., Alencar S. H. P., Gregory S. G., Matysse Collaboration 2019, MNRAS, 484, 5810

Horne K., 1986, PASP, 98, 609

Husser T. O., Wende-von Berg S., Dreizler S., Homeier D., Reiners A., Barman T., Hauschildt P. H., 2013, A\&A, 553, A6

Johansen A., 2009, in Strassmeier K. G., Kosovichev A. G., Beckman J. E., eds, IAU Symposium Vol. 259, Cosmic Magnetic Fields: From Planets, to Stars and Galaxies. pp 249-258 (arXiv: 0905.2597), doi:10.1017/S1743921309030592

Klein B., Donati J. F., 2019, MNRAS, 488, 5114

Klein B., Donati J. F., 2020, MNRAS, 493, L92

Königl A., 1991, ApJ, 370, L39

Kotani T., et al., 2014, in Proc. SPIE. p. 914714, doi:10.1117/12.2055075

Kotani T., et al., 2018, in Proc. SPIE. p. 1070211, doi: $10.1117 / 12.2311836$

Lanotte A. A., et al., 2014, A\&A, 572, A73

Lavail A., Kochukhov O., Wade G. A., 2018, MNRAS, 479, 4836

Lin D. N. C., Bodenheimer P., Richardson D. C., 1996, Nature, 380,606

Look I., Cavedoni C., Matsushige G., Vermeulen T., Elizares C., Bauman S., 2018, in Proc. SPIE. p. 107002U, doi:10.1117/12.2311409

Machado P., Widemann T., Luz D., Peralta J., 2014, Icarus, 243, 249

Machado P., Widemann T., Peralta J., Gonçalves R., Donati J.F., Luz D., 2017, Icarus, 285, 8

Mahadevan S., et al., 2014, The Habitable-zone Planet Finder: A status update on the development of a stabilized fiber-fed near-infrared spectrograph for the for the Hobby-Eberly telescope. p. $91471 \mathrm{G}$, doi: $10.1117 / 12.2056417$

Mann A. W., Feiden G. A., Gaidos E., Boyajian T., von Braun K., 2015, ApJ, 804, 64

Martioli E., et al., 2020, arXiv e-prints, p. arXiv:2006.13269

Maury A. J., et al., 2010, A\&A, 512, A40

Mayor M., 2019, in The La Silla Observatory - From the Inauguration to the Future. p. 16, doi:10.5281/zenodo.3245272

Mayor M., et al., 2003, The Messenger, 114, 20

Micheau Y., et al., 2012, in Ground-based and Airborne Instrumentation for Astronomy IV. p. 84462R, doi: $10.1117 / 12.926084$

Micheau Y., Bouyé M., Parisot J., Kouach D., 2015, in Techniques and Instrumentation for Detection of Exoplanets VII. p. $96051 \mathrm{Q}$, doi: $10.1117 / 12.2185188$

Micheau Y., et al., 2018, in Proc. SPIE. p. 107025R, doi:10.1117/12.2305937

Morin J., et al., 2008, MNRAS, 390, 567

Morin J., Donati J., Petit P., Delfosse X., Forveille T., Jardine M. M., 2010, MNRAS, 407, 2269
Morin J., Dormy E., Schrinner M., Donati J. F., 2011, MNRAS, 418, L133

Moutou C., et al., 2017, MNRAS, 472, 4563

Moutou C., et al., 2020, arXiv e-prints, p. arXiv:2008.05411

Muirhead P. S., et al., 2015, ApJ, 801, 18

Oliva E., Sanna N., Rainer M., Massi F., Tozzi A., Origlia L., 2018, in Proc. SPIE. p. 1070274, doi:10.1117/12.2309927

Parès L., et al., 2012, in Ground-based and Airborne Instrumentation for Astronomy IV. p. 84462E, doi:10.1117/12.925410

Pepe F., et al., 2013, The Messenger, 153, 6

Perruchot S., Hobson M., Bouchy F., Dolon F., Boisse I., Moreau F., Sottile R., Wildi F., 2018, in Proc. SPIE. p. 1070265, doi: $10.1117 / 12.2312057$

Probst R. A., et al., 2020, Nature Astronomy,

Pudritz R. E., Ray T. P., 2019, Frontiers in Astronomy and Space Sciences, 6, 54

Quirrenbach A., et al., 2012, CARMENES. I: instrument and survey overview. p. 84460R, doi:10.1117/12.925164

Rajpurohit A. S., Reylé C., Allard F., Homeier D., Schultheis M., Bessell M. S., Robin A. C., 2013, A\&A, 556, A15

Rajpurohit A. S., Allard F., Teixeira G. D. C., Homeier D., Rajpurohit S., Mousis O., 2018a, A\&A, 610, A19

Rajpurohit A. S., Allard F., Rajpurohit S., Sharma R., Teixeira G. D. C., Mousis O., Rajpurohit K., 2018b, A\&A, 620, A180

Rayner J., et al., 2016, iSHELL: a construction, assembly and testing. p. 990884, doi:10.1117/12.2232064

Reggiani H., Meléndez J., Yong D., Ramírez I., Asplund M., 2016, A\&A, 586, A67

Reiners A., Mrotzek N., Lemke U., Hinrichs J., Reinsch K., 2016, A\&A, 587, A65

Reshetov V., Herriot G., Thibault S., Désaulniers P., Saddlemyer L., Loop D., 2012, in Ground-based and Airborne Instrumentation for Astronomy IV. p. 84464E, doi:10.1117/12.927442

Ricker G. R., et al., 2016, The Transiting Exoplanet Survey Satellite. p. 99042B, doi:10.1117/12.2232071

Romanova M. M., Lovelace R. V. E., 2006, ApJ, 645, L73

Romanova M. M., Ustyugova G. V., Koldoba A. V., Lovelace R. V. E., 2004, ApJ, 616, L151

Romanova M. M., Long M., Lamb F. K., Kulkarni A. K., Donati J., 2011, MNRAS, 411, 915

Salz M., et al., 2018, A\&A, 620, A97

Schöfer P., et al., 2019, A\&A, 623, A44

Shu F. H., Galli D., Lizano S., Glassgold A. E., Diamond P. H., 2007, ApJ, 665, 535

Shulyak D., Reiners A., Engeln A., Malo L., Yadav R., Morin J., Kochukhov O., 2017, Nature Astronomy, 1, 0184

Smette A., et al., 2015, A\&A, 576, A77

Snellen I. A. G., de Kok R. J., de Mooij E. J. W., Albrecht S., 2010, Nature, 465, 1049

Snellen I., et al., 2015, A\&A, 576, A59

Sousa A. P., et al., 2016, A\&A, 586, A47

Strugarek A., Brun A. S., Matt S. P., Réville V., 2015, ApJ, 815, 111

Thibault S., Cui Q., Poirier M., Vallee P., Doyon R., Rabou P., Salmon D. A., 2003, Optical design of WIRCAM, the CFHT wide-field infrared camera. pp 932-943, doi:10.1117/12.460310

Thibault S., et al., 2012, in Ground-based and Airborne Instrumentation for Astronomy IV. p. 844630, doi:10.1117/12.926697

Tinetti G., et al., 2017, in European Planetary Science Congress. pp EPSC2017-713

Udry S., et al., 2019, A\&A, 622, A37

Ulmer-Moll S., Figueira P., Neal J. J., Santos N. C., Bonnefoy M., 2019, A\&A, 621, A79

Viana Almeida P., Melo C., Santos N. C., Figueira P., Sterzik M., Gameiro J. F., 2012, A\&A, 539, A62

Vidotto A. A., Donati J. F., 2017, A\&A, 602, A39 
Vidotto A. A., Jardine M., Morin J., Donati J.-F., Lang P., Russell A. J. B., 2013, A\&A, 557, A67

Vidotto A. A., Feeney N., Groh J. H., 2019, MNRAS, 488, 633

Villebrun F., et al., 2019, A\&A, 622, A72

Wildi F., Chazelas B., Pepe F., 2012, A passive cost-effective solution for the high accuracy wavelength calibration of radial velocity spectrographs. p. 84468E, doi:10.1117/12.926841

Yadav R. K., Christensen U. R., Morin J., Gastine T., Reiners A., Poppenhaeger K., Wolk S. J., 2015, ApJ, 813, L31

Yu L., et al., 2017, MNRAS, 467, 1342

Yu L., et al., 2019, MNRAS, 489, 5556

Zanni C., Ferreira J., 2013, A\&A, 550, A99

${ }^{1}$ Univ. de Toulouse, CNRS, IRAP, 14 av. Belin, 31400 Toulouse, France

${ }^{2}$ Univ. de Toulouse, CNRS, OMP, 14 av. Belin, 31400 Toulouse, France

${ }^{3}$ Observatoire du Mont-Mégantic, Département de physique de l'Université de Montréal, iREx, Montréal, Canada

${ }^{4}$ Univ. Grenoble Alpes, CNRS, IPAG, 38000 Grenoble, France

${ }^{5}$ Canada-France-Hawaii Telescope, Kamuela, Hawaii, USA

${ }^{6}$ Institut d'astrophysique de Paris, UMR7095 CNRS, Université Pierre \& Marie Curie, 98bis boulevard Arago, 75014 Paris, France

7 Obs. Astronomique de l'Université de Genève, Genève, Switzerland

${ }^{8}$ Aix Marseille University, CNRS, LAM, 13388 Marseille, France

${ }^{9}$ Hertzberg Institute of Astrophysics, National Research Council of Canada, Victoria, Canada

${ }^{10}$ Observatoire de Haute-Provence, CNRS, Aix Marseille University, Institut Pythéas, 04870 St Michel l'Observatoire, France

${ }^{11}$ Centre of Optics, Photonics and Lasers, Université Laval, Quebec, Canada

${ }^{12}$ Laboratório Nacional de Astrofísica, Itajuba, MG, Brazil

13 Institute of Astronomy \& Astrophysics Academia Sinica, Taipei, Taiwan

${ }^{14}$ European Southern Observatory, Vitacura, Santiago, Chile

15 Instituto de Astrofísica e Ciências do Espaço \& Faculdade de Ciências, Universidade do Porto, Porto, Portugal

${ }^{16}$ Dublin Institute for Advanced Studies, Dublin, Ireland

17 LUPM, Université de Montpellier, CNRS, Place E. Bataillon, 34095 Montpellier, France

18 Departamento de Fisica - ICEx - UFMG, Belo Horizonte, MG, Brazil

${ }^{19}$ Planétarium Rio Tinto Alcan, iREx, Montréal, Canada

20 Max-Planck-Institut für Quantenoptik, Garching, Germany; Menlo Systems GmbH, Martinsried, Germany

This paper has been typeset from a $\mathrm{T}_{\mathrm{E}} \mathrm{X} / \mathrm{LAT}_{\mathrm{E}} \mathrm{X}$ file prepared by the author. 